\title{
A Quantum Group Structure in Integrable Conformal Field Theories
}

\author{
Dirk-Jan Smit *
}

Institute for Theoretical Physics, University of Utrecht, P.O. Box 80.006, 3508 TA Utrecht, The Netherlands

\begin{abstract}
We discuss a quantization prescription of the conformal algebras of a class of $d=2$ conformal field theories which are integrable. We first give a geometrical construction of certain extensions of the classical Virasoro algebra, known as classical $W$ algebras, in which these algebras arise as the Lie algebra of the second Hamiltonian structure of a generalized Korteweg-de Vries hierarchy. This fact implies that the $W$ algebras, obtained as a reduction with respect to the nilpotent subalgebras of the Kac-Moody algebra, describe the integrability of a Toda field theory. Subsequently we determine the coadjoint operators of the $W$ algebras, and relate these to classical Yang-Baxter matrices. The quantization of these algebras can be carried out using the concept of a so-called quantum group. We derive the condition under which the representations of these quantum groups admit a Hilbert space completion by exploring the relation with the braid group. Then we consider a modification of the Miura transformation which we use to define a quantum $W$ algebra. This leads to an alternative interpretation of the coset construction for Kac-Moody algebras in terms of nonlinear integrable hierarchies. Subsequently we use the connection between the induced braid group representations and the representations of the mapping class group of Riemann surfaces to identify an action of the $W$ algebras on the moduli space of stable curves, and we give the invariants of this action. This provides a generalization of the situation for the Virasoro algebra, where such as invariant is given by the so-called Mumford form which describes the partition function of the bosonic string.
\end{abstract}

\section{Introduction and Summary}

One of the most prominent problems in string theory is to develop a prescription that incorporates both the geometric and algebraic concepts of its quantization.

\footnotetext{
* Address after October 1, 1989: Lawrence Berkeley Laboratory 1 Cyclotron road Berkeley, CA 94720, USA
} 
A first attempt in this direction, made a year ago by Friedan and Shenker [1], consists of a description of so-called modular geometries which can be defined on some infinite genus Riemann surface $\Sigma$. A modular geometry, which is an abstract description of a conformal field theory, is defined to be a bilinear functional on some noncommutative algebra $A_{c}$, labelled by a rational number $c$, which is invariant under the modular, or mapping class group $\pi_{1}(\Sigma)$.

The noncommutative algebra acts in the space of holomorphic sections of some vector bundle $L_{c}$ defined on the moduli space of stable Riemann surfaces of finite genus. The energy-momentum tensor of the corresponding conformal field theory is interpreted as a holomorphic flat connection on this bundle. If $L_{c}$ is a holomorphic line bundle it is, by a theorem of Harer [15], isomorphic to a power of the holomorphic line bundle of one-differentials. This has been the key point in the construction of Arbarello et al. [2], who proved that the cohomology of this space is isomorphic to the cohomology of the Virasoro algebra, using a description on a suitable infinite dimensional Grassmannian. As a result one finds that the infinitesimal geometry of the determinant line bundles on moduli space is described by the Virasoro algebra [10]. However, so far this formalism has been developed only for line bundle geometries. This is a serious problem since for full characterization of the string ground state it is necessary to incorporate all possible geometries for given $c$ such that the interplay between the representation theory of the conformal algebra and the geometry of the moduli space of (stable) vector bundles on Riemann surfaces is manifest.

A possible generalization is provided by the construction of so-called extended Virasoro or $W$ algebras which appeared in the generalization of the Sugawara construction to higher-order Casimir operators $[5,7,8,9]$. However the construction of these algebras as given by these authors does not provide a geometrical interpretation along the lines described above.

In the present paper we will give a geometrical construction of the classsical analogues of these $W$ algebras which is suitable for a quantization prescription that combines both its algebraic and geometrical properties. Our approach is connected to the Drinfeld-Sokolov theory [14] on bi-Hamilton (also called bi-Poisson) structures for integrable systems. As is well known, a characterizing property of an integrable conformal field theory is that the master equation is the classical Yang-Baxter equation. Recently much progress has been made in the understanding of the algebraic nature of this equation which culminated in a consistent quantization prescription in terms of a new algebraic object called a quantum group [11].

Before we proceed, let us give a simple argument why conformally invariant integrable field theories are necessarily of Wess-Zumino-Witten type, i.e. free field theories, or Toda field theories for some Lie group $G$ [9]. Consider the following general Ansatz for the action of a scalar field theory:

$$
S[\phi]=\frac{1}{2} \int d^{2} x\left(\partial \mu \phi^{i} \partial_{\mu} \phi^{j} g_{i j}+V(\phi)\right),
$$

where $g_{i j}$ is an arbitrary $n \times n$ matrix and $V(\phi)$ an arbitrary potential. It follows straight-forwardly using the classical field equations corresponding to (1.1), that the improved energy momentum tensor is traceless, provided that 


$$
V(\phi)=k \sum_{i} \exp g_{i j} \phi^{j}
$$

where $k$ is an integration constant. If one further requires that the theory be integrable (in the sense of [13]) it follows that $g_{i j}$ is the Cartan matrix of some simple Lie algebra $\mathscr{G}$. The theory defined in this way is known as a Toda field theory on the group manifold of some Lie group $G$. Note that (1.1) and $V$ as in (1.2), corresponds in the limit $k \rightarrow 0$ to a free field theory. To such a theory one may add a topological term, without affecting the integrability or the conformal invariance, thus turning it into a Wess-Zumino-Witten model. The conserved currents associated with (1.1) are known to build up a Kac-Moody algebra associated with $\mathscr{G}$.

In Sect. 2 we use some results of [14] to show that the classical conformal or $W$ algebra can be obtained from the Hamilton reduction of integrable nonlinear hierarchies associated with higher order differential operators. The space of differential operators we shall consider in this paper form the dual of an $S L_{N}$ Kac-Moody algebra. As is well known this space admits a natural Hamilton or Poisson structure. The space of differential operators endowed with this Hamilton structure, is identified as the phase space of the classical field theory. In addition to this natural Hamilton structure, there exista a second Hamilton structure, a fact which reflects a symmetry acting on this phase space. The pair of these Hamilton structures is called a bi-Hamilton structure.

The study of this structure is the central point in this section. One of the main properties we will discuss is the existence of a nonlinear map, which relates these Hamilton structures. Geometrically this corresponds to reducing the phase space with respect to this symmetry. This map is referred to as the Hamilton reduction. It is provided by the so-called Miura transformation. In the first part of this section we apply this formalism to quadratic differential operators to obtain the Virasoro algebra. This case is well known in the literature (see [17]). In the second part we generalize this to arbitrary differential operators of order $N$ and show that the resulting $W$ algebra contains terms of higher order in the generators, which can be considered as the classical analogues of the quadratic terms in the generalized sugawara construction in $[43,7,9]$. This approach was first followed by Fateev and Lykyanov [8], [48]. In the third part we present the relation of these algebras and a classical Yang-Baxter matrix by exploring the relation between the nonlinear hierarchies and the integrability of the Toda field theory. This provides an alternative characterization of $W$ algebra, namely as the Hamiltonian reduced associative free tensor algebra modulo the ideal generated by the Yang-Baxter relation. Such an algebra has the structure of what is called a bialgebra or Hopf algebra. This point of view is relevant for discussing a quantization of $W$ algebras.

In Sect. 3 we discuss a quantization description for the classical $W$ algebras formulated in terms of a quantum group built out of the classical Yang-Baxter matrix for the (classical) Toda field theory. This quantum group is essentially a generalization of the well known geometrical quantization procedure. It is defined as a deformation of the Hopf algebra structure described in Sect. 2. In this respect we would like to consider quantization of the classical reduced phase space as an irreducible representation of this quantum group. This is in general impossible, 
since the reduction in the classical situation corresponds to a nonlinear transformation which in general has no meaning after quantization. Or in other words, the second Hamilton structure on the reduced phase space does not define canonical conjugated variables. We will show in this section that this problem can be solved in terms of solving a condition on the representation content of a quantum group.

The section is divided into three parts. In the first part we derive the condition for which the representation of the relevant quantum group admits a Hilbert space completion, using a homomorphism of the quantum group into the braid group. Subsequently in Sect. 3.2 we introduce a normal ordering description in the Miura transformation which depends on the deformation parameter of the quantum group. Thus we are able to define a quantum $W$ algebra. Using the relation with the integrable nonlinear hierarchy we conclude that the effect of the Miura transformation is similar to adding a term to the energy momentum tensor of the underlying field theory corresponding to an electric charge "at infinity." As a result we find that application of the Miura transformation leads to the so-called coset construction for Kac-Moody algebras as developed in [6,7]. This relates the central charge of the $W$ algebras with the deformation parameter of the quantum group. Finally in Sect. 3.3 we explore the relation between certain representations of the braid group and the mapping class group to show that the quantized $W$ algebras define an action on the moduli space of stable curves. It is shown that the irreducible highest weight representations of a $W$ algebra define holomorphic flat connections on the moduli space. This provides a generalization of the result obtained in [2] for the Virasoro algebra, and shows that one may understand this action as an action of the underlying quantum group.

\section{Classical $\boldsymbol{W}$ Algebras}

In this section we will show that the Kac-Moody algebra acting as a symmetry algebra in a $S l_{N}$. Toda field theory admits a reduction which yields the classical extended Virasoro or $W$ algebras. As will be shown, these algebras are obtained as the Poisson algebra of the second Hamilton structure defined on the space of smooth differential operators. To introduce the formalism and to fix the notation we begin with briefly reviewing the situation for the Virasoro algebra, which is related to the second Hamilton structure of the Korteweg-de Vries equation. This is done in Sect. 2.1. Then, in Sect. 2.2 we generalize the formalism to arbitrary $A_{N}^{(1)} \mathrm{Kac}-$ Moody algebras using a theorem in [14]. In Sect. 2.3 we establish the connection with the classical Yang-Baxter $r$ matrix and the induced Lie bialgebra structure.

2.1 The bi-Hamilton Structure for the Virasoro Albebra. Our starting point is to relate the 2-cocycle on the infinite dimensional Virasoro algebra with a so-called bi-Hamilton structure. The technique we shall describe below is well known and originally due to Gervais and Kupershmidt $[16,17]$. We will generalize it later on.

The Virasoro algebra is generated by a symmetric 2-tensor, the energy momentum tensor of the underlying conformal field theory, which has Laurent 
expansion:

$$
T(z)=\sum_{-\infty}^{+\infty} L_{n} z^{-n-2}
$$

where

$$
L_{n}=\oint \frac{d z}{2 \pi i} z^{n+1} T(z) .
$$

As is well known the variation of $T(z)$ under a holomorphic change of coordinates $z \rightarrow z+w(z)$ is of the form:

$$
\delta T(z)=\frac{c}{12} w^{\prime \prime \prime}(z)+2 T(z) w^{\prime}(z)+T^{\prime}(z) w(z)
$$

where $c \in \mathbf{C}$ and the ' denotes $d / d z$. This is equivalent to the following Lie algebra relations for $L_{n}$ :

$$
\left[L_{n}, L_{m}\right]=(n-m) L_{n+m}+\frac{c}{12} n\left(n^{2}-1\right) \delta_{n+m}
$$

which is the defining relation for the Virasoro algebra.

Our aim is to show that (2.2) is the coadjoint operator related to the second Hamilton structure on the dual representation of the Virasoro algebra. To do so we will introduce two Hamilton operators associated with (2.3). Consider a ring $K$ of vectorfields $f(\theta)(d / d \theta)$ on the circle $S^{1}$ parametrized by $\theta$, which by virtue of a differential operator $\partial: K \rightarrow K$ is turned into a Lie algebra of $\operatorname{Diff}\left(S^{1}\right)$ :

$$
[f, g]=f g^{\prime}-f^{\prime} g \text {. }
$$

The Virasoro algebra is defined as the universal central extension by $\mathbf{C}$ described by the 2-cocycle [23]:

$$
\omega(f, g)=c \int_{0}^{2 \pi} f g^{\prime \prime \prime} d \theta, \quad c \in \mathbf{C},
$$

where $c$ is the central charge. Thus, $\omega$ defines a skewsymmetric form on (2.4), with $c$ belonging to its center. We shall write $(2.5)$ formally as

$$
\omega(f, g)=c f \partial^{3} g \text {. }
$$

Next, introduce the ring to $K$ denoted by $C[u]$ generated by a periodic $\mathbf{C}^{\infty}$-function $u(x)$ and all its derivatives. On $C[u]$ we have the induced derivation $\partial: u^{i} \rightarrow u^{i+1}$. Elements of the algebra in (2.4) will be called adjoint, whereas the elements of its dual (i.e. the Lie algebra on $C[u]$ induced by $\partial$ ) are called coadjoint. The Hamiltonian $H$ introduced above which is compatible with (2.4) is considered as an operator on $C[u]$, defined by

that is

$$
H=-(u,[f, g])=\int_{0}^{2 \pi} f(u \partial+\partial u) g,
$$

$$
H=u \partial+\partial u \text {. }
$$

We can introduce an operator $h_{\omega}$ on $C[u]$, corresponding to the 2-cocycle $\omega$ by

$$
h_{\omega}(f) g=\omega(f, g)
$$


so that

$$
h_{\omega}=c \partial^{3} .
$$

It is not difficult to show that $H_{2}=H+h_{\omega}$ is again a Hamilton operator on $C[u]$, which is associated with the Lie algebra (2.4) with the non-trivial 2-cocycle (2.5). Also the trivial cocyle on $C[u]$

$$
\lambda(f, g)=\frac{1}{2}(\partial f) g
$$

gives rise to a Hamilton operator $H_{1}=\frac{1}{2} \partial$ in an analogous way. The pair

$$
H_{1}=\frac{1}{2} \partial, \quad H_{2}=u \partial+\partial u+c \partial^{3}
$$

is called a bi-Hamilton structure [27]. Observe that (2.2) is of the form $\mathrm{H}_{2}$. For the moment we will put $c=\frac{1}{2}$. A well known fact states that to such bi-Hamilton structure there corresponds an integrable hierarchy of nonlinear evolution equations of the form:

$$
\partial_{t} u(x, t)=\operatorname{Pol}_{r}(u)
$$

where the evolution is taken in some time direction $t$. The right-hand side of (2.12) is a polynomial of degree $r$ in $C[u]$. In the case of (2.11), the associated hierarchy is known as the Korteweg de Vries (KdV) hierarchy, which has the generic form

$$
\partial_{t} u=H_{1}\left(\frac{\delta Q_{r+1}}{\delta u}\right)=H_{2}\left(\frac{\delta Q_{r}}{\delta u}\right),
$$

where $\delta / \delta u$ denotes the functional derivative with respect to $u$, and $Q_{r}$ denotes the $r^{\text {th }}$ conserved densities of the hierarchy, which is an element of $C[u]$. This first few are [27]:

$$
\begin{aligned}
& Q_{0}=\int \frac{u}{4} d x, \\
& Q_{1}=\int \frac{u^{2}}{4} d x, \\
& Q_{2}=\int\left(\frac{1}{2} u^{3}-\frac{1}{4}\left(u^{\prime}\right)^{2}\right) d x .
\end{aligned}
$$

Upon substituting these into (2.13) one finds the $\mathrm{KdV}$ equation as the first non-trivial element of the hierarchy

$$
\partial_{t} u=\frac{1}{4}\left(6 u u^{\prime}+u^{\prime \prime \prime}\right) \text {. }
$$

These Hamilton operators give rise to Poisson brackets by means of (2.15) and can be written as a Hamilton equation. Before we discuss the properties of these Poisson structures we consider the integrability of the $\mathrm{KdV}$ hierarchy a bit more in detail. The bi-Hamilton system (2.13) (with $c=\frac{1}{2}$ ) is closely related to a so-called Lax representation of the $\mathrm{KdV}$ hierarchy:

$$
\partial_{t} L=[L, A] \text {, }
$$

where $L$ is the Hill operator:

$$
L=\partial^{2}+u
$$


and $A$ the pseudo-differential operator of the form

$$
A=\left(L^{1 / 2}\right)_{+}^{r} \text {, }
$$

where $L^{1 / 2}$ is the pseudo-differential operator of the form

$$
L^{1 / 2}=\partial+\sum_{i=0}^{\infty} u_{i} \partial^{-i}
$$

such that $\left(L^{1 / 2}\right)^{2}=L$. This pseudo-differential operator is unique; the coefficients of it are differential polynomials in the coefficients of $L$. In (2.18) the + denotes the positive, i.e. the differential part. For $r=3 A$ is given by

$$
A=\left(L^{3 / 2}\right)_{+}=\partial^{3}+\frac{3}{2} u \partial+\frac{3}{4} u^{\prime},
$$

which upon substituting in (2.16) produces the $\mathrm{KdV}$ equation. The fact that this operator is of the same form as the second Hamilton operator has a nice interpretation which we shall discuss at the end of this subsection. For the moment we conclude that the Hamilton operators (2.11) define two Poisson structures on the space of functionals on the (smooth) manifold $M$ consisting of all operators of the form (2.17). These functionals are of the form

$$
\phi(L)=\int f\left(u(\xi), u^{1}(\xi), \ldots, u^{n}(\xi)\right) d \xi
$$

where $f$ is a polynomial in $C[u]$. The Poisson brackets induced on them are given by

$$
\{\phi, \psi\}_{i}=\int\left(\frac{\delta \phi}{\delta u} H_{i} \frac{\delta \psi}{\delta u}\right) d x
$$

For the functions $u(x)$ they are given by:

$$
\{u(x), u(y)\}_{i}=H_{i} \delta(x-y), \quad i=1,2
$$

with $H_{i}$ given in (2.11). So, we have found a relation between the non-trivial 2-cocycle of the Virasoro algebra and the bi-Hamilton structure of the KdV hierarchy [17].

We will now show that the Poisson structure induced by the second Hamilton operator defines the Virasoro Lie algebra on the dual space. For this it is convenient to reformulate the Lax equation (2.18) for $L$ in terms of a Lax equation for a linear differential operator $\mathscr{L}$ of the form:

where

$$
\mathscr{L}=\partial+q(x)+\Lambda .
$$

$$
q(x)=\left(\begin{array}{cc}
q_{11}(x) & q_{12}(x) \\
0 & q_{22}(x)
\end{array}\right), \quad \Lambda=\left(\begin{array}{ll}
0 & \lambda \\
1 & 0
\end{array}\right),
$$

in which $\lambda$ is the spectral parameter and the function $q(x)$ is an element of another differential ring $C[q]$ diffeomorphic to $C[u]$. It is important to realize that the form of $\mathscr{L}$ is unchanged under conjugation by constant matrices which have zeros on the diagonal. So the choice of $V$ is not unique.

It is easily seen that given $\mathscr{L}$ the operator $L$ is determined completely. That is, for each gauge the assignment $\mathscr{L} \rightarrow L$ is given by a gauge invariant differential 
polynomial relating $u$ with the functions $v$ in (2.25). The idea is very simple: one forms a quadratic polynomial in $\mathscr{L}$ whose coefficients are differential polynomials in $u$. Then, by imposing the eigenvalue equation for this quadratic differential operator (which is Hill's equation), the coefficients are fixed uniquely. In particular this will give a relation between the function $q(x)$ and $u(x)$ which is such that gauge equivalent operators $\mathscr{L}$ correspond to the same quadratic differential operator $L$. This differential polynomial in $u$ and $q$ is known as the Miura transformation. It is given by

$$
L=(\partial-q)(\partial+q) \Leftrightarrow u=q^{\prime}-q^{2} .
$$

Observe, that this transformation is not injective: knowing $L$ one finds $q(x)$ up to integration constants, which reflects the above mentioned gauge freedom. An important property of the differential operators $\mathscr{L}$ is that the time dependence of the functions $q(x)$ can be given as a Lax equation for $\mathscr{L}$,

$$
\partial_{t} \mathscr{L}=[\mathscr{A}, \mathscr{L}]
$$

If we require that under (2.26) this Lax equation goes over into (2.16) one finds that $\mathscr{A}$ is given by

$$
\mathscr{A}=\left(\begin{array}{cc}
q \lambda^{2}-\frac{1}{4}\left(2 q^{3}-q^{\prime \prime}\right) & \lambda^{3}-\frac{1}{2} \lambda\left(q^{2}+q^{\prime}\right) \\
\lambda^{3}-\frac{1}{2} \lambda\left(q^{2}+q^{\prime}\right) & -\lambda^{2} q+\frac{1}{4}\left(2 q^{3}-q^{\prime \prime}\right)
\end{array}\right),
$$

which upon substituting into (2.27) gives

$$
\partial_{t} q=\frac{1}{4}\left(q^{\prime \prime \prime}-6 q^{2} q^{\prime}\right)
$$

The equation is called the modified Korteweg-de Vries equation, corresponding to the modified Lax equation (2.27). We will discuss the relation between (2.16) and (2.27) in more detail in the next subsection. For the moment we simply observe that (2.28) can be expressed as

$$
\partial_{t} q=-\frac{1}{2} \partial\left(\frac{\delta Q_{1}}{\delta q}\right)_{u=q^{\prime}-q^{2}}
$$

That is, the operator

$$
H_{\text {mod }}=\frac{1}{2} \partial
$$

is a Hamilton operator for the modified $\mathrm{KdV}$ equation. This can be seen as a consequence from the fact that $H$ is a Hamilton operator for the $\mathrm{KdV}$ equation, and the identity

$$
\frac{1}{2} \partial^{3}+u \partial+\partial u=D\left(-\frac{1}{2} \partial\right) D^{*}
$$

where $D=\partial-2 q$ is the "Jacobian of $u$ with respect to $q$," and $D^{*}=-\partial-2 q$ its formal adjoint. We thus observe that the Hamilton structure defined by $H$ is obtained as a reduction of $H_{\text {mod }}$ [18].

We are now in the position to derive the classical Virasoro algebra from the second Hamilton structure by localizing the Miura transformation. To show that this operator indeed induces the Virasoro algebra we expand the functions $u(x)$ and $q(x)$ into Fourier series using techniques presented in [29]. We introduce the 
Fourier series $\mathscr{F}(u)$, respectively $\mathscr{F}(q)$ of a function $u(x)$ respectively $q(x)$ by

$$
\begin{aligned}
& u(x)=-\frac{12}{c} \sum_{i=-\infty}^{i=\infty} e^{-i n x} L_{n}+\frac{1}{2}, \\
& q(x)=-\frac{12}{c} \sum_{i=-\infty}^{i=\infty} e^{-i n x} a_{n},
\end{aligned}
$$

where we reintroduced the parameter $c$. Subsequently, we define the fourier series for the derivatives $u^{(j)}$ respectively $q^{(j)}$ as

$$
\begin{aligned}
& \mathscr{F}\left(u^{(j)}\right)=-\frac{12}{c} \sum_{n=-\infty}^{\infty}(-i n)^{j} e^{-i n x} L_{n}, \\
& \mathscr{F}\left(q^{(j)}\right)=-\frac{12}{c} \sum_{n=-\infty}^{\infty}(-i n)^{j} e^{-i n x} L_{n} .
\end{aligned}
$$

We refer to [29] (pages 32, 33) for the relevant statements on the calculus for these Fourier series, necessary to extend the fourier map $\mathscr{F}$ to the ring $C[u]$, respectively $C[q]$. After substituting into $(2.13,2.26,2.29)$ one obtains the corresponding equations for $L_{n}$ and $a_{n}$ :

$$
\begin{aligned}
\partial_{t} L_{n} & =\sum(n-m) L_{n+m} \frac{\delta Q_{1}}{\delta L_{m}}+\frac{c}{12} n\left(n^{2}-1\right) \frac{\delta Q_{1}}{\delta L_{-n}}, \\
\partial_{t} a_{n} & =-\frac{c}{12} n \frac{\delta Q_{1}}{\partial a_{-n}} \\
L_{n} & =-\frac{12}{c} \sum_{l+m=n} \frac{1}{2} a_{l} a_{m}+\frac{12}{c}(n-1) a_{n} .
\end{aligned}
$$

This gives the matrix components of the Hamilton operators:

$$
\begin{aligned}
\left(H_{\mathrm{mod}}\right)_{m n} & \sim-\frac{c}{12} n \delta_{n+m, 0}, \\
\left(H_{2}\right)_{m n} & =(n-m) L_{n+m}+\frac{c}{12} n\left(n^{2}-1\right) \delta_{m+n, 0},
\end{aligned}
$$

leading to the Poisson brackets:

$$
\begin{aligned}
& \left\{L_{n}, L_{m}\right\}=(n-m) L_{n+m}+\frac{c}{12} n\left(n^{2}-1\right) \delta_{n+m, 0}, \\
& \left\{a_{n}, a_{m}\right\}=\frac{c}{12} n \delta_{m+n, 0} .
\end{aligned}
$$

This shows that the second Hamilton operator of the modified $\mathrm{KdV}$ induces a classical representation of the Virasoro algebra on the dual space. Thus the classical Virasoro algebra is obtained as the reduction of a free oscillator algebra which is homogeneous in $n$. We will see in Sect. 3 that this is a general property, and is in fact related to the $S L_{2}$ Kac-Moody algebra. 
Now that we have obtained the Virasoro algebra we will discuss its coadjoint operator. To find this operator we use some results of the coadjoint orbit method as presented in [26]. Using the invariant bilinear form on the virasoro algebra one can embed the space $M$ of Hill operators into the dual space denoted by Vir*:

$$
L=\partial^{2}+u \rightarrow(u, c=-1) \in \operatorname{Vir}^{*},
$$

where we used the notation as in [26]. Using the Poisson brackets of the Virasoro algebra we find the coadjoint operator

$$
\operatorname{ad}_{f, c}^{*}(u,-1)=\left(\partial^{3}+u \partial+\partial u\right) f
$$

This is in agreement with the fact that $L$ transforms covariantly under a Diff $S^{1}$ transformation $x \rightarrow \theta(x)$ :

$$
L \rightarrow\left(\theta^{\prime}\right)^{-3 / 2} \tilde{L}\left(\theta^{\prime}\right)^{-1 / 2}
$$

where

$$
\begin{aligned}
& \tilde{L}=\partial^{2}+\tilde{u}, \\
& \tilde{u}=\left(\theta^{\prime}\right)^{2} u(\theta(x))+\frac{1}{2}\left(\frac{\theta^{\prime \prime \prime}}{\theta^{\prime}}-\frac{3}{2}\left(\frac{\theta^{\prime \prime}}{\theta^{\prime}}\right)^{2}\right) .
\end{aligned}
$$

This shows that the function $u(x)$ transforms as an energy momentum tensor $T$. In this respect one may view the Lax equation for $L$ corresponding to the $\mathrm{KdV}$ equation as a conformal deformation by the third order differential operator $A$. In Sect. 3 we will discuss a quantization of the coadjoint operator (2.43) in which we will relate this deformation with the operator product expansion of the energy momentum tensor.

We conclude this section with a brief comment on the classification of the coadjoint representations [24,23,25], which follows by determining the kernel of (2.43), i.e. by classifying all $f \in$ Vir which for given $u$ solve the equation

$$
\delta u=f^{\prime \prime \prime}+2 f^{\prime} u+u^{\prime} f=0 .
$$

From this it is clear that the orbits fall roughly into two classes depending on whether $f$ contains zeros or not. If $f$ contains no zeros it follows that the coadjoint orbit contains the constant vector $u=u_{0}$. The orbits containing a constant coadjoint vector are known to be $\operatorname{Diff} S^{1} / \operatorname{Rot} S^{1}$, and $\operatorname{Diff} S^{1} / S L_{2}(\mathbb{R})^{n}$, where the index $n$ denotes the copy of $S L_{N}$ generated by $L_{ \pm n}, L_{0}$. It is not difficult to show that a critical point of the "Hamiltonian" $L_{0}$ in (2.38) precisely corresponds to orbits containing the constant coadjoint vector. Further as can be seen from (2.38), only $\operatorname{diff} S^{1} / \operatorname{Rot} S^{1}$, and $\operatorname{diff} S^{1} / S L_{2}(\mathbb{R})^{n}$ for $n=1$ the critical points are stable. For these orbits $L_{0}$ is bounded from below, hence will lead to highest weight representations after quantization in a semi-classical expansion. For the case $n>1$ there will also exist highest weight representations, but these will in general not be irreducible. In section three we will discuss a quantization description for the Poisson brackets (2.41-42) (including the case $n>1$ ), i.e. for the coadjoint orbits for which the Hamiltonian has critical points. 
2.2 The Geometrical Construction of the $W$ Algebras. In this section we will generalize the construction outlined in the previous section by explaining the bi-Hamilton structure and its relation with the modified Lax equation using an abstract theorem of Drinfeld and Sokolov. To do so, and to discuss its consequences we need some more machinery to deal with arbitrary differential operators, as was developed in $[27,28]$. We will consider differential operators $L$ of the form:

$$
L=\partial^{N}+\sum_{i=0}^{N-2} u_{i} \partial^{i}
$$

where $u_{i}=u_{i}^{(0)} \in C^{\infty}\left(S^{1}, \mathbf{C}\right)$, the zero ${ }^{\text {th }}$ order component of the ring $C[u]$, consisting of functions $u_{i}$ and all their derivatives. Furthermore we denote by $M$ the smooth manifold of all operators $L$ of the form (2.44).

We now introduce the pseudo-differential symbol $X$ as

$$
X=\sum_{i=0}^{N-2} a_{i} \partial^{-i}
$$

so that $X$ is in fact an integral symbol. Denote by $F$ the space of all functionals of the form

$$
\dot{\phi}(L)=\int f\left(u_{0}(\xi), \ldots, u_{N-1}(\xi), \ldots, u_{0}^{(n)}(\xi), \ldots, u_{N-1}^{(n)}(\xi)\right) d \xi .
$$

To each pseudo-differential operator $X$ we associate a functional $l_{X}: M \rightarrow \mathrm{C}$ by

$$
l_{X}=\operatorname{Tr} X L
$$

where

$$
\operatorname{Tr} X=\int \operatorname{res} X=\int a_{-1}(x) d x .
$$

We will use this formalism to study the Lie algebra structures induced by the Hamilton operators corresponding to some generalized Lax equation. One can show [27] that associated with each operator $L$ there exists a Lax equation of the form:

$$
\partial_{t} L=[L, A]
$$

where $A$ is defined as $a=\sum_{i=0}^{m} c_{i}\left(L^{m / N}\right)_{+}$with $c_{i}$ arbitrary complex constants. The left-hand side of (2.49) is a differential operator of order not exceeding $n-2$, while the right-hand side is in general of order $m+N-2$, which makes that there are $m$ relations between the coefficients of $A$ and $L$. These relations determine the operator $A$ in terms of $L$. The pseudo-differential operator $L^{1 / N}$ is of the form (2.19) and satisfies the condition $\left(L^{1 / N}\right)^{N}=L$. The Lax equation (2.49) can be given in terms of a bi-Hamilton structure which induce poisson brackets of the form (2.22-23). The Poisson brakcets can be computed with the following

Theorem 2.1. ([14)] Let $\phi, \psi$ be two linear affine functionals defined on the manifold $M$ of differential operators $L$ of the form (2.45). Denote by $X_{\phi}$ respectively $X_{\psi}$ the 
pseudo differential operators corresponding to $\phi$ respectively $\psi$,

$$
X_{\phi}=\sum_{i=1}^{N-1} \partial^{-i} \frac{\delta \phi}{\delta u_{i-1}} \quad X_{\psi}=\sum_{i=1}^{N-1} \partial^{-i} \frac{\delta \psi}{\delta u_{i-1}},
$$

where $\delta / \delta u$ denotes the variational derivative with respectively to $u$.

1. On $F$ there can be defined a bi-Poisson structure given by

$$
\begin{aligned}
& \{\phi, \psi\}_{1}(L)=\operatorname{Tr}\left(\left[L, X_{\phi}\right]_{+} X_{\psi}\right), \\
& \{\phi, \psi\}_{2}(L)=\operatorname{Tr}\left(\nabla_{X_{\phi}}(L) X_{\psi}\right) .
\end{aligned}
$$

where $\nabla_{X_{\phi}}(L)$ is defined as

$$
\nabla_{X_{\phi}}(L) \equiv L\left(X_{\phi} L\right)_{+}-\left(L X_{\phi}\right)_{+} L .
$$

2. The Lax equation (2.49) is Hamiltonian with respect to both Poisson structures, and for a given operator $A$, (as in (2.49)), the Hamiltonian function is given by $H_{m}(L)=N \operatorname{Tr}\left(L^{m / N}\right) / m$.

It is worthwhile to mention that the operator $\nabla_{X_{\phi}}$ behaves under an arbitrary constant translation of the operator $L \rightarrow L+k$ as

$$
\nabla_{X_{\phi}}(L) \rightarrow \nabla_{X_{\phi}}(L)-k\left[L, X_{\phi}\right]_{+} .
$$

Later we shall see that this defines a Hamilton structure also. Observe that the theorem generalizes the bi-Hamilton structure for the Korteweg-de Vries equation to differential operators of order $N$. In particular the conserved charges follow from the formula given in the second part of the theorem.

In the rest of this section we will give a Lie algebraic interpretation for both of the Hamilton structures in the context of conformal field theory. More precisely, we will show that the first Hamilton structure can be interpreted as the natural Hamilton structure on the coadjoint orbits of an affine Kac-Moody algebra. We will use the formalism as presented in ref. [30]. The application of the results in [30] to classical $W$-algebras has been discussed independently by I. Bakas in [50]. The second Hamilton structure will turn out to define Poisson brackets of generalizations of the Virasoro algebra known as $W$-algebras. The two Hamilton structures are related via a non-linear mapping known as the Miura transformation. As we will discuss, this provides a 'classical' version of the Sugawara construction for Kac-Moody algebras. In addition to this we shall find that the Poisson manifold for the second Hamilton structure has a 'hidden' gauge symmetry which stems form the Kac-Moody algebra associated with the first Hamilton structure. In order to make these ideas explicit, we use results of the Drinfeld-Sokolov theory on the relation between affine Kac-Moody algebras and Lax equations for first order differential operators.

We start with introducing the space $\mathscr{M}$ of all first order differential operators of the form

$$
\mathscr{L}=\partial+V(x)
$$

where $V(x)=V_{a}(x) T^{a}$, and $T^{a}$ denote the generators of a simple Lie algebra $\mathscr{G}$, 
with commutation relations

$$
\left[T^{a}, T^{b}\right]=f_{c}^{a b} T^{c}
$$

It is well known [30] that on $\mathscr{M}$ there is an action of the loop group $L G=C^{\infty}\left(S_{1}, G\right)$, with $G$ the Lie group associated with $\mathscr{G}$. Under $g(x) \in L G$ we have

$$
\mathscr{L}^{\prime}=g^{-1}(x) \mathscr{L} g(x)=\partial+g^{-1}(x) V(x) g(x)+g^{-1}(x) d g(x),
$$

which shows that $L G$ acts via gauge transformations. We recall that there is a natural central extension, which in terms of the algebra $L \mathscr{G}$, is given by

$$
\omega\left(V_{1}, V_{2}\right)=\int d x\left\langle V_{1}(x), V_{2}(x)\right\rangle,
$$

where $\langle$,$\rangle denotes the non-degenerate G$-invariant bilinear form on $L \mathscr{G}$. The central extension of $L \mathscr{G}$ is denoted by $\widehat{L \mathscr{G}}$. Using this bilinear form one may identify $L \mathscr{G}$ with the smooth part of its dual space $L \mathscr{G}^{*}$, and in this respect the action of the loop group $L G$ (without central extension), can be considered as describing the coadjoint action of $L G$ in the this dual space $\widehat{L G}^{*}$.

Now, it is well known that on $L \mathscr{G}^{*}$ there is a natural Hamilton structure. Denoting by $\phi(V)$ and $\psi(V)$ two functionals on $L \mathscr{G}$, then the Poisson bracket corresponding to this structure is given as

$$
\{\phi(V(x)), \psi(V(y))\}=\int d \xi\left\langle\left[\frac{\delta \phi(V(\xi))}{\delta V(\xi)}, \frac{\delta \psi(V(y))}{\delta V(y)}\right], \mathscr{L}\right\rangle .
$$

It is easily verified using the properties of the bilinear form

$$
\begin{aligned}
\langle u,[v, w]\rangle & =-\langle v,[u, w]\rangle, \\
\left\langle u, v^{\prime}\right\rangle & =-\left\langle v, u^{\prime}\right\rangle,
\end{aligned}
$$

that this bracket satisfies the Jacobi identity. Furthermore, if we take $V=V^{a}$ one recovers the Poisson relations for the affine algebra $L \mathscr{G}$ :

$$
\left\{V_{a}(x), V_{b}(y)\right\}=f_{a b}^{c} V_{c} \partial_{x} \delta(x-y)-\frac{1}{2} \delta_{a b} \partial_{x} \delta(x-y),
$$

where we used the normalization $\operatorname{Tr}\left(T^{a} T^{b}\right)=-\frac{1}{2} \delta^{a b}$.

In fact, this bracket has an important property namely, it still satisfies the Jacobi identity if we replace the operator $\mathscr{L}$ by $a$ the operator

$$
\tilde{\mathscr{L}} \equiv \lambda K+\mu \mathscr{L}
$$

with $K$ a constant matrix. In this way one obtains from (2.58) a bi-structure on $L \mathscr{G}^{*}$, by taking either $\lambda=0$ or $\mu=0$ : (see [30] and e.g. [50])

$$
\begin{aligned}
& \{\phi(V(x)), \psi(V(y))\}_{1}=\int d \xi\left\langle\left[\frac{\delta \phi(V(\xi))}{\delta V(\xi)}, \frac{\delta \psi(V(y))}{\delta V(y)}\right], K\right\rangle, \\
& \{\phi(V(x)), \psi(V(y))\}_{2}=\int d \xi\left\langle\left[\frac{\delta \phi(V(\xi))}{\delta V(\xi)}, \frac{\delta \psi(V(y))}{\delta V(y)}\right], \partial+V(x)\right\rangle .
\end{aligned}
$$

In other words one finds that $\{\}=,\lambda\{,\}_{1}+\mu\{,\}_{2}$ again defines a Hamilton structure. This Hamilton structure leads to the relations for the loop algebra $\widehat{L \mathscr{G}}$, 
with $V=V^{a}$ :

$$
\left\{V^{a}(x), V^{b}(y)\right\}=\lambda f_{c}^{a b} V^{c}(x) \partial_{x} \delta(x-y)-\frac{1}{2} \mu \delta^{a b} \partial_{x} \delta(x-y) .
$$

In the case where $\mu=0$ the Hamilton structure describes on action similar to the action of the (finite dimensional) Lie group $G$ on the coadjoint representations of its algebra. One calls this Poisson bracket also the Kirillov bracket.

Note that on the Poisson manifold defined by (2.58) we have the loop group $L G$ acting by gauge transformations. In order to relate the Hamilton structures (2.61-62) with the bi-Hamilton structures on the space $F$ one has to isolate this action. This can be done by specifying a gauge. For this it turns out to be useful to consider operators.

$$
\mathscr{L}=\partial+q(x)+\Lambda
$$

where $q(x)$ is an $N \times N$-matrix upper triangular matrix:

and $\Lambda$ is the $N \times N$-matrix

$$
q(x)=\left(\begin{array}{cccc}
q_{11}(x) & \cdots & \cdots & q_{1 N}(x) \\
0 & q_{22}(x) & & \vdots \\
\vdots & & \ddots & \vdots \\
0 & \cdots & \cdots & q_{N N}(x)
\end{array}\right)
$$

$$
\Lambda=\left(\begin{array}{ccccc}
0 & \cdots & \cdots & \cdots & \lambda \\
1 & 0 & & & 0 \\
0 & 1 & \ddots & & \vdots \\
\vdots & & & \ddots & \\
0 & \cdots & \cdots & 1 & 0
\end{array}\right),
$$

with $\lambda$ the spectral parameter. Formulae $(2.65-66)$ are direct generalizations of (2.24-25). For this operator $\mathscr{L}$ the gauge transformations correspond to conjugation with unipotent matrices, (whose entries are functions of $x$ ). It follows that one can choose in each gauge equivalence class a representative denoted by $\mathscr{L}^{\text {can }}$ of the form

$$
\mathscr{L}^{c a n}=\partial+q^{c a n}+\Lambda
$$

where

$$
q^{c a n}(x)=\left(\begin{array}{ccc}
0 & \cdots & q_{1}^{c a n}(x) \\
\vdots & & \vdots \\
0 & \cdots & q_{N}^{c a n}(x)
\end{array}\right) .
$$

The relation between the matrix elements of $q$ and $q^{\text {can }}$ follows from the operator equation

$$
\operatorname{det} \mathscr{L}=\operatorname{det} \mathscr{L}^{\text {can }} \text {. }
$$

One of the main results in [14] is that the map $\mathscr{L} \rightarrow \mathscr{L}^{\text {can }}$ is a Poisson morphism 
for the bracket (2.62). The map $\mathscr{L} \rightarrow \mathscr{L}^{c a n}$ is referred to as the Miura transformation. It can be computed explicitly as follows. Recall the argument made in the previous section, by which we assigned to each gauge equivalence operator $\mathscr{L}$ a quadratic differential operator $L$. It is straightforward to verify that the same argument applies also to higher order differential operators. We use this to compute the Miura transformation $\mu: \tilde{\mathscr{M}} \rightarrow M$ explicitly:

$$
L=\prod_{i=1}^{N}\left(\partial-q_{i}^{c a n}\right) \Leftrightarrow u_{i-1}=-q_{i}^{c a n} .
$$

We are now in a position to relate the bi-structure in (3.2.64-65) for $G \simeq G L_{N}$ with the bi-Hamilton structures on functionals on the space $M$ of operators $L$ defined in theorem 2.1. Denote by $\tilde{\mathscr{M}}$ the space of operators $\mathscr{L}$ of the form (2.64), and by $\tilde{\mathscr{F}}$ the space of functionals on $\tilde{\mathscr{M}}$. The Hamilton structure on the space $\tilde{\mathscr{F}}$ is obtained from the Hamilton structure (2.58) on $\mathscr{F}$ by applying the Miura transformation $\mathscr{L} \rightarrow \mathscr{L}^{\text {can }}$. By virtue of (2.70) one finds that this Poisson structure can be expressed in terms of functionals on $F$ :

$$
\{\tilde{\phi}(u(x)), \tilde{\psi}(u(y))\}=\int \operatorname{res}\left(\nabla_{X_{\phi}}(L) X_{\psi}\right),
$$

where we used the notation explained earlier, which is precisely the form of the second Hamilton structure introduced in theorem 2.1.

Summarizing, we have shown that, upon using the Miura transformation, the Hamilton structure (2.58) for the affine loop algebra $G L_{N}$ defines the second Hamilton structure (2.51) on the space $F$ of functionals on the affine space $M$ of operators $L$. Furthermore, it follows that any linear combination of the two Hamilton structures on $F$ again defines a Hamilton structure on $F$. One refers to the Poisson algebras associated with $(2.51)$ as the Gelfand-Dickey algebra $\mathrm{GD}\left(G L_{N}\right)$. We may consider this combined Hamilton structure on $F$ as formally describing the coadjoint action on the dual algebra. This generalizes the construction of the previous section which lead to the Virasoro algebra. In fact by imposing suitable conditions on the form of the operator $L$ one may obtain Gelfand-Dickey algebras for the classical Lie algebras.

Let us conclude this section with discussing the algebra $\operatorname{GD}\left(S L_{N}\right)$. This algebra is obtained by imposing the condition that the coefficient $u_{n-1}=0$. For consistency it turns out that one should modify the definition of $X_{\phi}$ as

$$
X_{\phi}=\sum_{i=1}^{N-1} \partial^{-i} \frac{\delta \phi}{\delta u_{i-1}}+\partial^{-N} b_{N}
$$

where the coefficient $b_{N}$ is determined by the condition that $\operatorname{res}\left[L, X_{\phi}\right]=0$. This is precisely the term of degree $N-1$ in the definition of $\nabla_{X_{\dot{\phi}}}$.

We will now work out the second Poisson structure and the localization of the Miura transformation for the case $S L_{3}$, which we will identify as the classical analogue of the spin-3 algebra found by Zamolodchikov [5]. We start from the third-order differential operator

$$
L=\partial^{3}+\partial u+u \partial+v=\partial^{3}+2 u \partial+v+u^{\prime}
$$

It transforms covariantly under a reparametrization of the circle $S^{1}$. 
This operator gives rise to the so-called Boussinesq equation,

which can be written as

$$
\partial_{t}^{2} u=\frac{1}{3} u^{\prime \prime \prime}+\frac{4}{3}\left(u^{2}\right)^{\prime \prime},
$$

$$
\partial_{t} u=v^{\prime}, \quad \partial_{t} v=\frac{1}{3}+\frac{8}{3} u u^{\prime} .
$$

The first and second Hamilton operator as computed from Theorem 2.1 are $H_{1}=\frac{1}{2} \partial$,

$H_{2}=\left(\begin{array}{cc}\frac{1}{2} \partial^{3}+\partial u+u \partial & v \partial+2 \partial v \\ v \partial+2 \partial v & \frac{1}{3} \partial^{5}+10 u \partial^{3}+15 u^{\prime} \partial^{2}+9 u^{\prime \prime} \partial+16 u^{2} \partial+2 u^{\prime \prime \prime}+16 u u^{\prime}\end{array}\right)$,

with the associated densities

$$
\begin{aligned}
& Q_{1}=\int\left(-\frac{1}{3}\left(u^{\prime}\right)^{2}+\frac{8}{9} u^{3}+\frac{1}{2} v^{2}\right) d x, \\
& Q_{0}=\int \frac{1}{2} v d x .
\end{aligned}
$$

Correspondingly, the Poisson structure induced by $\mathrm{H}_{2}$ will split into four parts. In order to find them in the canonical form we expand the functions $u$ and $v$ in fourier components. For $u$ we take the expansion (2.34), while for $v$ the appropriate expansion reads:

$$
v(x)=\frac{12}{c} \sqrt{10} \sum_{n=-\infty}^{\infty} e^{i n x} W_{n} .
$$

Then one finds that the $L_{n}$ generate the Virasoro algebra while the other parts in (2.76) give rise to the Poisson structures

$$
\begin{aligned}
i\left\{L_{n}, W_{n}\right\}= & (2 n-m) W_{n+m}, \quad \text { plus the complex conjugated bracket, } \\
i\left\{W_{n}, W_{m}\right\}= & (n-m)\left(\frac{1}{15}(n+m+2)(n+m+3)+\frac{1}{6}(n+2)(m+2)\right) L_{n+m} \\
& +\frac{16}{22+5 c}(n-m) \Lambda_{n+m}+\frac{c}{360} n\left(n^{2}-1\right)\left(n^{2}-4\right) \delta_{n+m, 0},
\end{aligned}
$$

where

$$
\Lambda_{n}=\sum_{m=-\infty}^{+\infty} L_{n-m} L_{m}
$$

This last bracket does not define a Lie algebraic structure in the usual sense since it contains terms quadratic in the generators. We can consider (2.79) as the classical analogue of the algebra constructed by Zamolodchikov in [5]. Upon localizing the Miura transformation one obtains an oscillator representation of the $W$ algebra in a similar way as in the previous section, without normal ordering.

Observe also that the Virasoro algebra is contained in the $W$ algebra of $S l_{3}$. In fact this is a consequence of the following natural filtration of the $W$ algebras. Define the subspace $F_{i}$ as

$$
f_{i}=\left\{X=\sum_{i=0}^{N-2} \partial^{-i} x_{i} \in F \mid x_{1}=x_{2}=\cdots=x_{N-i-2}=0\right\} \text {. }
$$


Then one checks easily that

$$
\left[F_{i}, F_{j}\right] \subset F_{i+j}
$$

thus implying the filtration

$$
F_{0} \subset F_{1} \subset \cdots \subset F_{n-2}=W\left(S l_{N}\right)
$$

In particular we observe that the Virasoro is contained in $F_{0}$.

2.3 The Relation with Toda Field Theory and the Classical Yang-Baxter Equation. Now that we have constructed the $W$ algebras and discussed their representations, we will discuss the relation with the Toda field theory and with the classical Yang-Baxter equation (YBE). This amounts to giving a Lie algebraic interpretation for the modified Lax equation and the associated nonlinear hierarchy. For this we have to introduce some notation.

Let $\mathscr{G}$ be a rank $r$ Kac-Moody algebra, with canonical generators $\left(e_{i}, f_{i}, h_{i}\right)$. Denote by $\mathscr{G}_{-}, \mathscr{G}_{0}, \mathscr{G}_{+}$the subalgebras generated by the $f_{i}, h_{i}, e_{i}$ respectively. Let $\overline{\mathscr{G}}$ be the loop algebra, obtained by dividing out the center from $\mathscr{G}$. In the principal grading we set $\mathscr{G}_{0}=\mathscr{H}$, the Cartan subalgebra. Let $\mathscr{S}^{ \pm}$denote the positive respectively negative component of the principal Heisenberg algebra $\mathscr{S}$ with respect to this grading of $\mathscr{G}$. Furthermore we let $\left\{\lambda_{i}\right\}$ be a basis of $\mathscr{S}$, where the index runs over the exponents of $\mathscr{G}$.

Next, we fix a vertex $c_{k}$ of the Dynkin diagram of $\mathscr{G}$. Then we can consider the so-called homogeneous grading of $\mathscr{G}$ specified by the requirement that $f_{k} \in \mathscr{G}_{-}$, $e_{k} \in \mathscr{G}_{+}$and all other generators belong to $\mathscr{G}_{0}$. Thus the Dynkin diagram of the finite dimensional algebra $\mathscr{G}_{0}$ is obtained from $\mathscr{G}$ by removing the vertex $c_{k}$. Let $\mathscr{B}^{ \pm}$denote the two Borel subalgebras associated to $\mathscr{G}_{0}$ and denote by $\mathscr{N}$ the nilpotent subalgebra associated with $\mathscr{B}$.

The following theorem gives an algebraic interpretation for the modified Lax equation.

Theorem 2.4. Let $\mathscr{L}=\partial+\Lambda+q$, where $\Lambda=\lambda_{1}=\Sigma e_{i}$, and where $q$ is a matrix function of $x$ in the set of upper triangular matrices, $\mathscr{B}^{+}$. Then there are unique elements $U \in[\Lambda, \overline{\mathscr{G}}]^{-}$and $H \in \overline{\mathscr{S}}^{-}$such that

$$
e^{\operatorname{ad} U} \mathscr{L}=\partial+\Lambda+H \text {. }
$$

For a proof we refer to [14, Prop. 6.2].

For us the following, easily verified consequence is very important.

Theorem 2.5. Let $U$ be defined as above and set $\mathscr{A}(\lambda)_{+}=e^{-\mathrm{ad} U}(\lambda)$, where only the component of positive degree is taken. If $q$ is a matrix in $\mathscr{B}$ respectively, $\mathscr{H}$ then the Lax equation

$$
\partial_{t} \mathscr{L}=\left[\mathscr{A}(\lambda)_{+}, \mathscr{L}\right]
$$

is also defined on $\mathscr{B}$ respectively $\mathscr{H}$.

With this theorem we can now define the generalized $\mathrm{KdV}$ and modified $\mathrm{KdV}$ hierarchies associated with $\mathscr{G}$ as 
Definition 2.2. The modified $\mathrm{KdV}$ hierarchy associated with $\mathscr{G}$ is defined by (2.84) with $V$ belonging to the Cartan subalgebra $\mathscr{H}$ of the Kac-Moody algebra $\mathscr{G}$ in the principal grading.

To obtain the $\mathrm{KdV}$ hierarchy we have to remove the gauge freedom in the operator $\mathscr{L}$ with $q \in \mathscr{H}$. Namely it is seen that conjugation of $\mathscr{L}$ with unipotent matrices corresponding to $\mathscr{N}$ leaves the form of $\mathscr{L}$ invariant. So in order to define the $\mathrm{KdV}$ hierarchy one has to show that $(2.85)$ is covariant with respect to gauge transformations of the form $\exp (\operatorname{ad} n(x))$ with $n(x) \in \mathscr{N}$. This is shown in [14] [Sect. 6], and will not be explained here.

Denote by $\bar{q}$ a gauge equivalence class in the set of matrices $\mathscr{B}^{-}$. One defines the $\mathrm{KdV}$ hierarchy associated with $\mathscr{G}_{0}$ by

$$
\partial_{t} \bar{q}=\left[\mathscr{A}\left(\lambda_{i}\right)_{+}, \partial+\Lambda+\bar{q}\right] .
$$

Now, any class $\bar{q}$ has a representing element $q$ in the Cartan algebra $\mathscr{H}$ which satisfies (2.86). The map $q \rightarrow \bar{q}$ is the Miura transformation for $\hat{\mathscr{G}}$.

The connection with the Toda field theory can be made as follows. The field $\phi$ is taken in the Cartan subalgebra $\overline{\mathscr{H}}$ of $\overline{\mathscr{G}}$. The two dimensional field equation reads

$$
\frac{\partial^{2} \phi}{\partial \tau \partial x}=\sum_{i=0}^{r} e^{\alpha_{i}(\phi)} \bar{h}_{i},
$$

which upon expanding $\phi=\sum_{i=0}^{r} u_{i} \bar{h}^{i}$ yields

$$
\frac{\partial^{2} u_{i}}{\partial x \partial \tau}=\exp \left(\sum_{j=0}^{r} a_{i j} u_{j}\right),
$$

where $a_{i j}$ denotes the Cartan matrix of $\overline{\mathscr{G}}$. As is well known, this equation can be obtained from a so-called zero curvature condition

$$
[\mathscr{L}, \tilde{\mathscr{L}}]=0,
$$

where

$$
\begin{aligned}
& \mathscr{L}=e^{-\phi} \partial_{x} e^{\phi}+\Lambda=\partial_{x}+\phi^{\prime}+\Lambda, \\
& \overline{\mathscr{L}}=\partial_{\tau}+e^{a d(\phi)} \tilde{\Lambda}
\end{aligned}
$$

in which $\Lambda=\sum e_{i}$ and $\tilde{\Lambda}=\sum f_{i}$. It is not hard to verify that (2.88) gives rise to two Lax equations, one for $\mathscr{L}$ and one for $\tilde{\mathscr{L}}$. If we set $\phi^{\prime}=q$ then $\mathscr{L}$ gives rise to a generalized modified $\mathrm{KdV}$ hierarchy.

One concludes that $\phi$ and $\partial_{x} \phi$ or $\phi$ and $\partial_{t} \phi$ are canonically conjugate with respect to the appropriate Poisson bracket

$$
\left\{\partial \phi_{i}(x), \phi_{j}(y)\right\}=\delta_{i j} \delta(x-y) .
$$

This bracket is known as the fundamental Poisson bracket. It defines a classical $r$ matrix, satisfying the classical Yang-Baxter equation (YBE) as follows. For any $\mathscr{G}$ valued function $T$ the bracket gives rise to a matrix $r \in \wedge^{2} \mathscr{G}$ satisfying:

$$
\left\{T_{1}, T_{2}\right\}=\left[r_{12} \otimes T_{1} \otimes T_{2}\right] \text {. }
$$


Upon substituting this into the fundamental Poisson brackets one finds that the matrix $r$ defines an ad $\mathscr{G}$ invariant element $\langle r, r\rangle \in \wedge^{3} \mathscr{G}$ defined as:

$$
\langle r, r\rangle=\left[r_{12}, r_{13}\right]+\left[r_{12}, r_{23}\right]+\left[r_{13}, r_{23}\right],
$$

where $\langle$,$\rangle denotes the Schouten bracket [12]. If the left-hand side is zero then$ (2.92) is the classical YBE. IN [12] it is shown that a classical $r$ matrix satisfying (2.92) defines on $\mathscr{G}$ a Lie bi-algebra structure. A Lie bialgebra consists of a Lie algebra $\mathscr{G}$ together with a linear map:

$$
\psi: \mathscr{G} \rightarrow \wedge^{2} \mathscr{G}
$$

which satisfies two conditions. First, it must be 1 -cocyle with values in the $\mathscr{G}$ module $\wedge\left(\mathscr{G} \otimes \mathscr{G}^{*}\right)$, that is, for $x, y \in \mathscr{G}$ it satisfies:

$$
[x, \psi(y)]-[y, \psi(x)]=\psi([x, y]) .
$$

Secondly, the dual map $\psi^{*}$ which is a map

$$
\psi^{*}: \wedge^{2} \mathscr{G}^{*} \rightarrow \mathscr{G}^{*}
$$

must induce a Lie algebra structure on $\mathscr{G}^{*}$, which means that $\psi^{*}$ satisfies the Jacobi identity. The cocycle condition on $\psi$ means that $\psi$ defines an element in the cohomology group $H^{1}\left(\mathscr{G}, \wedge^{2} \mathscr{G}\right)$. In our case the underlying finite dimensional Lie algebra is simple so that $H^{1}\left(\mathscr{G}, \wedge\left(\mathscr{G} \otimes \mathscr{G}^{*}\right)\right)=0$. Hence for all $x \in \mathscr{G}$ there exists an element $r$ such that

$$
\psi(x)=[x, r] .
$$

One can verify easily that $\psi^{*}$ satisfies the Jacobi identity if and only if $r$ satisfies (2.93). We will denote such a bialgebra by $(\mathscr{G}, \partial r)$. Evaluated on the generators for $\mathscr{G}$ one finds that the 1 -cocycle $\psi$ is given by:

$$
\psi\left(h_{i}\right)=0, \quad \psi\left(e_{i}\right)=e_{i}=e_{i} \wedge h_{i}, \quad \psi\left(f_{i}\right)=f_{i} \wedge h_{i}
$$

Now suppose that $r$ is invertible. Then its inverse $r^{-1}$ defines an element of $\wedge^{2} \mathscr{G}^{*}$, which is in fact a linear functional

$$
r^{-1}: \wedge^{2} \mathscr{G}^{*} \rightarrow \mathbf{R}
$$

It follows that when $r$ is invertible it determines an ad $\mathscr{G}$ invariant symplectic structure on $\mathscr{G}$, hence $r$ itself defines a nondegenerate Poisson bracket on $\mathscr{G}^{*}$. This is in particular the case for the Poisson bracket (2.91) by which we could choose naturally conjugated variables in the Toda field theory.

The Poisson brackets that correspond to the $\mathrm{KdV}$ and the modified $\mathrm{KdV}$ hierarchies contain derivatives, which makes that they define "degenerate" Poisson structures, in the sense that there is no obvious choice for variables that are conjugated to each other. These Poisson brackets correspond with $r$ matrices which cannot be invertible. Even if the $r$ matrix is not invertible it can be shown that the classical Yang-Baxter equation amounts to a Lie bialgebra. Furthermore, one can define the "quantization" of these Poisson brackets in terms of "quantum groups". This seems the right context to discuss the quantization of the Poisson brackets for the $W$ algebras. In the next section we will discuss such a quantization scheme. 


\section{Quantizing $W$ Algebras Using Quantum Groups}

In the present section we will discuss a quantization prescription for the $W$ algebras obtained in the previous section which can be viewed as a combinaton of the geometrical quantization scheme, or "quantization of orbits" [26], and a quantization of functions on Lie groups. This section is divided into three parts. In the first we discuss the quantization of the classical Lie bialgebra structure induced by the classical $r$ matrix leading to so-called quantum groups and we will derive a theorem on the unitary representations of these quantum groups. Then in part two we define the quantum $W$ algebra by introducing so-called $\tau$ functions and a normal ordering in the Miura transformation. Finally in part three we discuss the action of the $W$ algebra on the moduli space of stable Riemann surfaces.

3.1 The Hilbert Space Completion of a Quantum Group. As we have seen, the quantization of a solution of the classical YBE amounts to a quantization prescription of the Lie bialgebra structure induced by this solution. Such quantization has recently been described by Drinfeld. Rather than giving a detailed analysis of this description, we summarize some relevant facts here that we will use later one. We refer to $[11,31,32]$ for a more rigorous treatment.

The Lie bialgebras we are interested in are of the form (2.92). Using elements of formal group theory one can show that such a Lie bialgebra actually has the form of a universal enveloping algebra denoted by $U(\mathscr{G})$. The quantization of a Lie bialgebra is then defined as a one dimensional deformation of this universal enveloping algebra with deformation parameter $q$. The deformed universal enveloping algebra will be denoted by $U_{q}(\mathscr{G})$. An important condition is that the deformation must be such that infinitesimally it is isomorphic to the original Lie bialgebra. Drinfeld showed that this requirement means that $U_{q}$ is generated by the operator valued matrix functions $T_{i}$ generating a non-commutative and non-commutative Hopf algebra which he called a quantum group.

To make this more precise we start with the following definition:

Definition 3.1. Let $V=\sum \lambda^{i} W_{i}, W_{i} \simeq \mathbf{C}^{N}$ and let $t_{i j}(\lambda)=\sum\left(t_{i j}\right)_{m} \lambda^{m}$, where $\left(t_{i j}\right)_{m} \in$ End $\left(W_{m}\right)$, a complex $N \times N$ matrix and $\lambda$ the rapidity or spectral parameter defining the grading of the infinite dimensional $\mathbf{Z}$ graded vector space $V$. Furthermore, introduce a matrix $R(\lambda, \mu) \in \operatorname{End}(V \otimes V)$, satisfying the following equation in End $(V \otimes V \otimes V):$

$$
R_{12}(\lambda, \mu) R_{13}(\lambda, v) R_{23}(\mu, v)=R_{23}(\mu, v) R_{13}(\lambda, v) R_{12}(\lambda, \mu) .
$$

The quantum group $A_{R}$ is defined as the free tensor algebra generated by the $t_{i j}(\lambda)$ modulo the ideal generated by

where

$$
R_{12}(\lambda, \mu) T_{1}(\lambda) T_{2}(\mu)=T_{2}(\mu) T_{1}(\lambda) R_{12}(\lambda, \mu),
$$

$$
T_{1}(\lambda)=T(\lambda) \otimes \mathrm{id}, \quad T_{2}(\lambda)=\mathrm{id} \otimes T(\lambda), \quad T(\lambda)=\sum T_{m} \lambda^{m}, \quad T_{m}=\left(t_{i j}\right)_{m} \in \operatorname{End}\left(W_{m}\right) .
$$

Equations (3.1) and (3.2) are called the quantum YBE and the fundamental commutation relation respectively. Observe that (3.2) implies that the family of operators generated by $\operatorname{Tr} T(\lambda)$ ( $\operatorname{Tr}$ denoting the trace), is commuting so that (3.2) 
can be regarded as the condition that the quantum theory be integrable. If $R$ allows a perturbation expansion of the form:

$$
R(\lambda, \mu)=1+i \hbar r(\lambda, \mu)+\mathcal{O}\left(\hbar^{2}\right),
$$

then the relations reduce in the "classical limit" $q \rightarrow 1$ to (2.91-92).

From (3.2) it follows that if $T_{1}(\lambda)$ and $T_{2}(\lambda)$ are two representations of $A_{R}$ then the product $T_{1}(\lambda) T_{2}(\lambda)$ is also a representation. In a more abstract way this implies the existence of an algebra homomorphism, called the comultiplication $\Delta$ :

$$
\Delta: A_{R} \rightarrow A_{R} \otimes A_{R}
$$

acting on the generators $t_{i j}(\lambda)$ as:

$$
\Delta\left(t_{i j}(\lambda)\right)=\sum_{k=1}^{n} t_{i k}(\lambda) \otimes t_{k j}(\lambda)
$$

There are two important automorphisms of the algebra $A_{R}$ that we have not discussed yet. Although we will not make use of them in the sequel, we present them for convenience.

Theorem 3.1. The algebra $A_{R}$ generated by $T$ has the following automorphisms:

1. Then antipode $S$, acting on $T$ as

$$
S(T(\lambda))=\left(T^{-1}(\lambda)\right)^{t}
$$

2. The inversion $I$ :

$$
I(T(\lambda))=T^{-1}(-\lambda) .
$$

These properties give the quantum group $A_{R}$ the structure of a Hopf algebra.

One can introduce the dual space to the algebra $A_{R}$, as $\operatorname{Hom}\left(A_{R}, \mathbf{C}\right)$. It is clear that this is an associative algebra and the multiplication on it is induced by the comultiplication on $A_{R}$. So in $\operatorname{Hom}\left(A_{R}, \mathbf{C}\right)$ there exists a co-unit $\mathscr{E}$ such that

$$
\mathscr{E} t_{i j}=\delta_{i j}
$$

For some values of $q$ the representation space of a quantum group has the interpretation of a Hilbert space of states of a quantum field theory. The dual representation has therefore the interpretation of the space of observables of the theory. The non-cocommutativity of the dual is thus crucial to describe noncommuting observables. To determine the values for $q$ for which the quantum group has this interpretation we first have to study some aspects of the representations of the $q$ deformed algebra. Starting from the $A_{N}^{(1)}$ Kac-Moody algebra in the principal grading with Cartan matrix $a_{i j}$ and $k_{i}=q^{h_{i}}$ the following representation of the dual of the quantum group is known $[19,11]$ :

$$
\begin{gathered}
k_{i} k_{i}^{-1}=k_{i}^{-1} k_{i}=1, \quad k_{i} k_{j}=k_{j} k_{i}, \quad k_{i} e_{j} k_{i}^{-1}=q^{a_{i j}} e_{j}, \quad k_{i} f_{j} k_{i}^{-1}=q^{-a_{i j}} f_{j}, \\
{\left[e_{i}, f_{j}\right]=\delta_{i j} \frac{k_{i}-k_{i}^{-1}}{q^{2}-q^{-2}}, \quad e_{i}^{2} e_{i \pm 1}-\left(q^{2}-q^{-2}\right) e_{i} e_{i \pm 1} e_{i}+e_{i \pm 1} e_{i}^{2}=0} \\
f_{i}^{2} f_{i \pm 1}-\left(q^{2}-q^{-2}\right) f_{i} f_{i \pm 1} f_{i}+f_{i \pm 1} f_{i}^{2}=0 .
\end{gathered}
$$


For convenience we also present the representation of the comultiplication, there co-unit and the antipode:

$$
\begin{aligned}
& \Delta\left(e_{i}\right)=e_{i} \otimes k_{i}^{-1}+k_{i} \otimes e_{i}, \quad \Delta\left(f_{i}\right)=f_{i} \otimes k_{i}^{-1}+k_{i} \otimes f_{i}, \quad \Delta\left(k_{i}\right)=k_{i} \otimes k_{i}, \\
& \mathscr{E}\left(e_{i}\right)=0=\mathscr{E}\left(f_{i}\right), \quad \mathscr{E}\left(k_{i}\right)=1, \quad S\left(e_{i}\right)=-q e_{i}, \quad S\left(f_{i}\right)=-q f_{i}, \quad S\left(k_{i}\right)=k_{i}^{-1} .
\end{aligned}
$$

This representation can be considered as a $q$-deformation of the Cartan-Weyl basis of the Kac-Moody algebra $A_{N}^{(1)}$. Indeed for $q \rightarrow 1$ the relations (3.9) go over into the defining relations of the "classical" Kac-Moody algebra with Cartan matrix $a_{i j}$. After the quantization of the Lie bialgebra structure of the fundamental Poisson bracket (2.85) is replaced by the fundamental commutation relation (3.2).

It is easily verified that $U_{q}$ has in addition to $\Delta$ a second comultiplication, which is defined by

$$
\Delta^{\prime}=\sigma^{\circ} \Delta
$$

where $\sigma: U_{q} \otimes U_{q} \rightarrow U_{q} \otimes U_{q}$ denotes the permutation, $\sigma(a \otimes b)=b \otimes a$.

A particular interesting situation is when the $R$ matrix is invertible. In that case the comultiplication $\Delta^{\prime}$ is related to the inverse $R^{-1}$ by

$$
\Delta^{\prime}=R \Delta R^{-1} \text {. }
$$

In Sect. 2 we discussed the Lie bialgebra structure $(\mathscr{G}, \partial r)$ in terms of the classical $r$ matrix. We conclude from the discussion given there that the cocomutator in $\mathscr{G}$ given by

$$
x \rightarrow[x \otimes \mathbf{I d}+\mathbf{I d} \otimes x, r]
$$

is an algebra homomorphism. We want to have a similar statement for the quantum group $U_{q}$ in terms of the matrix $R$. It is not difficult to see that if

$$
(\Delta \otimes \mathrm{Id}) R=R_{13} R_{23}, \quad(\mathrm{Id} \otimes \Delta) R=R_{13} R_{12},
$$

then the map $l \mapsto(l \otimes \mathbf{I d})(R)$ which is a map $U_{q} \rightarrow A_{R}$ is an algebra homomorphism. If an element $R \in U_{q} \otimes U_{q}$ satisfies $(3.12,14)$ then $R$ satisfies automatically the Yang-Baxter equation (3.1) for constant spectral parameters. The quantum group $U_{q}$ is called in this case a quasi-triangular Hopf algebra. The $R$ matrix for (3.9) as found by Jimbo [19] satisfies (3.12-14).

The representation (3.9) has a crucial property which relates its structure with the representation theory of the so called Hecke algebra. This fact turns out to be very important in the discussion of the irreducible unitary representations of $U_{q}$. To discuss this relation we have to introduce some more notation. Let $V$ be a finite dimensional vector space over $\mathbf{C}$ of dimension $N$, with basis $E_{1}, E_{2}, \ldots, E_{N}$. Le $R=\mathbf{C}\left[q^{1 / 2}, q^{-1 / 2}\right]$, and form $V_{R}=V \otimes_{\mathrm{C}} R$. Define

$$
v^{(p)} \equiv \underbrace{V \otimes_{\mathbf{C}} \cdots \otimes_{\mathbf{C}} \cdots \otimes_{\mathbf{C}} V}_{n \text {-times }}
$$

and

$$
v_{R}=v^{(n)} \otimes_{C} R
$$


If $n=1$ this is a module for $U_{q}$. The generators of the algebra $U_{q}$ act on it according to

$$
\begin{array}{ll}
e_{i} E_{a}=\delta_{i a} E_{a+1} & e_{i} E_{n}=0 \\
f_{i} E_{a}=\delta_{i+1 a} E_{a-1} & f_{i} E_{1}=0 \\
k_{i} E_{a} & =q^{\left(\delta_{i+1 a}-\delta_{i a) / 2}\right.} E_{a} .
\end{array}
$$

Because of the fact that the comultiplication on $U_{q}$ is an algebra homomorphism, we conclude that $v_{R}$ is a module for $U_{q}$ for arbitrary $n$. In the sequel we need the action of $U_{q}$ on $v_{R}$ for $n=2$. It reads

$$
\begin{aligned}
& e_{i}\left(E_{a} \otimes E_{b}\right)=\delta_{i a} q^{\left(\delta_{i b}-\delta_{i+1, b}\right) / 2} E_{a+1} \otimes E_{b}+\delta_{i b} q^{-\left(-\delta_{i a}-\delta_{i+1, a) / 2}\right.} E_{a} \otimes E_{b+1} \\
& f_{i}\left(E_{a} \otimes E_{b}\right)=\delta_{i+1, a} q^{\left(\delta_{i b}-\delta_{i+1, b) / 2}\right.} E_{a-1} \otimes E_{b}+\delta_{i+1, b} q^{-\left(\delta_{i a}-\delta_{i+1, a) / 2}\right.} E_{a} \otimes E_{b-1} \\
& k_{i}\left(E_{a} \otimes E_{b}\right)=q^{\left(\delta_{i+1, a}-\delta_{i a}+\delta_{i+1, b}-\delta_{i b}\right) / 2} E_{a} \otimes E_{b} .
\end{aligned}
$$

One can define an operation $T: v_{R} \rightarrow v_{R}$, for arbitrary $n$ which is the $q$-analogue of the symmetric group. For $p=n$ it reads

$$
T\left(E_{a} \otimes E_{b}\right)=E_{b} \otimes E_{a}+\left(q^{-1}-q^{\langle a, b\rangle}\right) E_{a} \otimes E_{b},
$$

where

$$
\langle a, b\rangle=\left\{\begin{array}{rll}
-1 & \text { if } & a>b \\
0 & \text { if } & a=b \\
1 & \text { if } & a<b .
\end{array}\right.
$$

Theorem 3.2. The commutant of the algebra $U_{q}$ in $\operatorname{End}(V)$ is the Hecke algebra $\mathscr{H}_{n}(q)$, and the $U_{q}$ module generated by $\left(e_{i}, f_{i}, k_{i}\right)$ is completely reducible.

Outline of Proof. It is straightforward to show that the action of $T$ commutes with the action of $U_{q}$ on $v_{R}$. For this it suffices to take $n=2$, and use the above explicit formulae. For $n=3$ one may write action of $T$ as follows. Let us denote $E_{a} \otimes E_{b} \otimes E_{c} \equiv E_{a b c}$. Then

$$
\begin{aligned}
& T_{1} E_{a b c}=\left\{\begin{array}{lll}
E_{a b c} & \text { if } & a>b \\
q^{-1} E_{b a c} & \text { if } & a=b \\
E_{b a c}+\left(q^{-1}+q\right) E_{a b c} & \text { if } & a<b
\end{array}\right. \\
& T_{2} E_{a b c}=\left\{\begin{array}{lll}
E_{a c b} & \text { if } & b>c \\
q^{-1} E_{a c b} & \text { if } & b=c \\
E_{a c b}+\left(q^{-1}+q\right) E_{a b c} & \text { if } & b<c .
\end{array}\right.
\end{aligned}
$$

This gives two identities:

$$
\begin{gathered}
T_{1} T_{2} T_{1}=T_{2} T_{1} T_{2}, \\
\left(T_{i}-q^{-1}\right)\left(T_{i}+q\right)=0 .
\end{gathered}
$$

Considering this in for arbitrary $p$ one gets in this way $n-1$ generators $T_{i}$ which 
staisfy:

$$
\begin{aligned}
& T_{i} T_{i+1} T_{i}=t_{i+1} T_{i} T_{i+1} \quad \text { if } \quad|i-j| \leqq 2 \\
& T_{i} T_{j}=T_{j} T_{i} \quad \text { if } \quad|i-j|>2 \\
& \left(T_{i}-q^{-1}\right)\left(T_{i}+q\right)=0 \text {. }
\end{aligned}
$$

The algebra generated by the $T_{i}$ is called the Hecke algebra of the symmetric group, denoted by $\mathscr{H}_{n}$.

This shows that the Hecke algebra is contained in the commutant of $U_{q}$. To show that the Hecke algebra forms the whole commutant, we will study the limit $q \rightarrow 1$, and show that the dimensions of the commutants do not "jump" in this limit. For $q$ indeterminate we know that the representation theory of the Hecke algebra generated by $T_{i}$ is the same as the representation theory of the symmetric group. Now we let $K=\mathbf{C}\left(q^{1 / 2}\right)$, the field of rational functions in $q^{1 / 2}$. We define

$$
\begin{aligned}
& \mathscr{L}_{R}=\text { the } R \text {-subalgebra of End } u_{q}\left(v_{R}\right) \text { generated by } e_{i}, f_{i}, k_{i} \\
& \mathscr{L}_{K}=\text { the } K \text {-subalgebra of } \operatorname{End}_{K}\left(v_{K}\right) \text { generated by } e_{i}, f_{i}, k_{i} \\
& \mathscr{L}_{\mathrm{C}}=\text { the } \mathrm{C} \text {-subalgebra of } \operatorname{End}_{\mathrm{C}}\left(v_{\mathrm{C}}\right) \text { generated by } \bar{e}_{i}, \bar{f}_{i}, \bar{k}_{i}=1 .
\end{aligned}
$$

Correspondingly, we define by

$$
\begin{aligned}
& \mathscr{J}_{R}=\text { the } R \text {-subalgebra of } \operatorname{End}_{R}\left(v_{R}\right) \text { generated by } T_{i} \\
& \mathscr{J}_{K}=\text { the } K \text {-subalgebra of } \operatorname{End}_{K}\left(v_{K}\right) \text { generated by } T_{i} \\
& \mathscr{J}_{C}=\text { the } \mathbf{C} \text {-subalgebra of } \operatorname{End}_{C}\left(v_{C}\right) \text { generated by } \bar{T}_{i}=s_{i} .
\end{aligned}
$$

In the limit $q \rightarrow 1$ we have $R \rightarrow \mathbf{C},\left(e_{i}, f_{i}, k_{i}\right) \rightarrow\left(\bar{e}_{i}, \bar{f}_{i}, 1\right)$ and $T_{i} \rightarrow s_{i}$.

Now it is not hard to show that the commutant of $\mathscr{L}_{c}$ is precisely $\mathscr{J}_{c}$. Furthermore, $\mathscr{J}_{C}$ is isomorphic to the group algebra of the symmetric group. Since the symmetric group is a finite group it follows from a theorem of Maschke that the $\mathscr{J}_{C}$ is a semisimple algebra. The map $\mathscr{L}_{R} \rightarrow \mathscr{L}_{C}$ is surjective. Hence, one can find elements $y_{1}, y_{2}, \ldots, y_{n}$ such that for all $i \bar{y}_{i}=x_{i}$ and these may serve as a basis in $\mathscr{L}_{c}$. It is not difficult to show from this that the elements $y_{i}$ are in fact linearly independent over $K$. This implies that

$$
\operatorname{dim}_{\boldsymbol{K}} \mathscr{L}_{\boldsymbol{K}} \geqq \operatorname{dim}_{\mathbf{C}} \mathscr{L}_{\boldsymbol{C}}
$$

Denote by $C_{K}$ the commutant of $\mathscr{J}_{K}$ in $\operatorname{End}_{K}\left(v_{K}\right)$, and by $C_{C}$ the commutant of $\mathscr{J}_{C}$ in $\operatorname{End}_{C}\left(v_{C}\right)$. The next step is to show that

$$
\operatorname{dim}_{K} C_{K}<\operatorname{dim}_{C} C_{C}
$$

Consider $\mathcal{O} \equiv \operatorname{End}_{R}\left(v_{R}\right)$. This is an $R$-submodule of $\operatorname{End}_{K}\left(v_{K}\right)$. One has the following exact sequence for $R$ modules:

$$
0 \rightarrow \mathcal{O} \cap C_{K} \rightarrow \mathcal{O} \rightarrow \mathcal{O} /\left(\mathcal{O} \cap C_{K}\right) \rightarrow 0
$$

It now follows that $\mathcal{O} \cap C_{K}$ is a direct summand of $\mathcal{O}$. (We leave the verification 
of this to the reader). Using this one shows that if $f \in \mathcal{O} \cap C_{K}$ then

$$
f T_{i}=T_{i} f \Leftrightarrow \bar{f} T_{i}=\bar{f} s_{i}=s_{i} \bar{f} .
$$

Now in the limit $q \rightarrow 1$ we have $\mathcal{O} \rightarrow \operatorname{End}_{C}\left(v_{C}\right)$, and it now follows that

$$
\operatorname{dim}_{C} \overline{\mathcal{O} \cap \mathscr{C}_{K}}=\operatorname{dim}_{K} C_{K}
$$

hence

$$
\operatorname{dim}_{\boldsymbol{C}} \overline{\mathcal{O} \cap C_{K}} \leqq \operatorname{dim} C_{C}
$$

which in turn implies $\operatorname{dim}_{K} C_{K} \leqq \operatorname{dim}_{C} \mathscr{L}_{C}$. Since $\mathscr{L}_{K} \subset C_{K}$. We now conclude that $\operatorname{dim}_{K} \mathscr{L}_{K} \leqq \operatorname{dim}_{K} C_{K}$. Combining this with the result above one concludes

$$
\operatorname{dim}_{K} \mathscr{L}_{K}=\operatorname{dim}_{C} \mathscr{L}_{C}
$$

and the commutant is indeed the Hecke algebra. A short argument shows that $\mathscr{L}_{K}$ and $\mathscr{J}_{K}$ are semi-simple algebras over $K$. As a result we find that the module $v_{K}$ is completely reducible.

Now, let $U$ be a $U_{q}$ module. One defines spaces $V_{\omega}, \omega \in \mathbf{C}^{N}$, by

$$
V_{\omega}=\left\{x \in U \mid k_{i} x=\omega_{i} x, \quad i=1, \ldots, N\right\},
$$

where $\omega=\left(\omega_{1}, \ldots, \omega_{N}\right), \omega_{i} \in \mathbf{C}$. This is called the weight space corresponding to the weight $\omega$. Let $U^{-}$be the subalgebra of $U_{q}$ generated by the $f_{i}$. One defines a highest weight module $U$, by the property that $U=A \cdot x$ for an $x \in V_{\omega}$. $\omega$ is called the highest wieght of $U$. It has not difficult to show that for any $\omega$ there exists a highest weight module with highest weight given by $\omega$.

Now let $U_{K}$ be the $U_{q}$ algebra over $K$ generated by all the $\left(e_{i}, f_{i}, k_{i}^{ \pm 1}\right), i=1, \ldots, N$. Denote by $U_{\bar{K}}$ its closure. Let $U_{R}$ be the $R$-subalgebra of $U_{q}$ generated by $f_{i}^{(n)}=f_{i}^{n} /[n] !, e_{i}^{(n)}=e_{i}^{n} /[n] !, k_{i}^{ \pm 1}$, where $i=1, \ldots, N, n=1,2, \ldots$. The notation is as in [19]:

$$
[n] ! \equiv \frac{q-q^{-1}}{q-q^{-1}} \frac{q^{-2}-q^{-2}}{q-q^{-1}} \cdots \frac{q^{n}-q^{-n}}{q-q^{-1}} .
$$

This is the $q$-analogue of the usual $n$-factorial formula.

Suppose we are given an irreducible $U_{\bar{K}}$-module $V$ finite dimensional over $\bar{K}$. Then because the module is completely reducible, we may write it as

$$
V=\oplus_{\omega} V_{\omega} .
$$

We want to show that the module $V$ is given by $V=V_{R} \otimes \bar{K}$, where $V_{R}$ is an $R$-submodule of $V$. We will not prove this in full detail but show the steps of the reasoning. One may take the module $V_{R}$ to be given by the $A_{R}$-subalgebra, denoted by $A_{R}^{-}$generated by all the $f_{i}^{(n)}$ :

$$
V_{R}=A_{R}^{-} v \subset V
$$


where $v \in V$ is a vector $(\neq 0)$ such that $e_{i} v=0$. After some combinatorics of $q$-numbers one concludes that the module $V_{R}$ is a finitely generated $R$-module. From that it follows from a rather straightforward but lengthy argument that $V$ is indeed given by (3.32).

This fact is useful to study the limit limit $R \rightarrow$ C, i.e. $q^{1 / 2} \mapsto 1$. One can show that in this limit

$$
\operatorname{dim}_{C} \bar{V}=\operatorname{dim}_{\bar{K}} V
$$

where $\bar{V}=V_{R} \otimes_{R} \mathrm{C}$, and it follows that the generators of generate in this limit the enveloping algebra of a Kac-Moody algebra. This is made more precise in the following theorem:

Theorem 3.3. Any integrable highest weight module $U_{0}$ of (the universal enveloping algebra of) the Kac-Moody algebra underlying $U_{q}$, admits a deformation, i.e. there exists a highest weight module $U$ of $U_{q}$ such that when $q \rightarrow 1$ it tends to $U_{0}$. The dimensions of the weight spaces of $U_{q}$ are given as " $q$ numbers" of the dimensions of corresponding weight spaces of $U_{0}$ which are given by the Weyl-Kac character formula.

This result was already announced in the paper by Jimbo [19].

We may thus conclude that for generic $q$ the representation theory of the Hopf algebra $A$ is very much the same $a$ for Kac-Moody algebras. In particular one can apply without difficulty the theory on unitary highest weight modules.

The situation changes when we consider the case where $q$ is a root of unity. This case bears some resemblance with the theory of subfactors of representations, in particular with the work of Wenzl [21]. For generic $q$ the representations $\mathscr{H}_{n}(q)$ are classified by Young diagrams, like for the symmetric group. If $\lambda=\left[\lambda_{1} \cdots \lambda_{k}\right]$ is a Young diagram with $n$ boxes; $\lambda_{1}$ boxes in the first row, $\lambda_{2}$ boxes in the second etc., then we can associate each irreducible representation of $\mathscr{H}_{n}(q)$ with a $\lambda$. When $q$ is an $l$-th root of unity, most of these representations are singular. In [21] the regular representations have been determined in this case. The conclusion is that only diagrams with at most $k$ rows, where $k \leqq l+1$ and $\lambda_{1}-\lambda_{k} \leqq l-k$ provide regular representations of $\mathscr{H}_{n}(q)$ where $q^{l}=1$. It is clear that for a Hilbert space completion of the representations of the quantum group we have to restrict to the regular representations. So we conclude that the commutant of the quantum group as computed in theorem (3.2) in the case $q$ is a root of unity is given as a quotient of the Hecke algebra, corresponding to the regular representations of $\mathscr{H}_{n}(q)$. That is, we find a restriction on the representation content of a quantum group. In the next section we will come back to this phenomenon.

We conclude this section with discussing a property of the algebra which we will use in the following sections. The algebra $U_{q}$ turns out to be intimately related with the braid group of $n$ strings. More precisely we will show

Theorem 3.4. Let $U_{q}$ be generated by $\left(e_{i}, f_{i}, k_{i}\right)$ satisfying the relations (3.9). For each $i=1, \ldots, N$ there exists a unique algebra homomorphism $\theta_{i}: U_{q} \rightarrow U_{q}$ acting on the generators of $U_{q}$ according to: 


$$
\begin{aligned}
& \begin{cases}\theta_{i} e_{i}=f_{i} k_{i}^{-2} & \\
\theta_{i} e_{j}=q^{-1} e_{j} e_{i}-q e_{i} e_{j} & \text { if } a_{i j}=-1 \\
\theta_{i} e_{j}=e_{j} & \text { if } a_{i j}=0\end{cases} \\
& \left\{\begin{array}{lll}
\theta_{i} f_{i}=k_{i}^{-2} e_{i} & & \\
\theta_{i} f_{j}=q f_{j} f_{i}-q^{-1} f_{i} f_{j} & \text { if } & a_{i j}=-1 \\
\theta_{i} f_{j}=f_{j} & \text { if } a_{i j}=0
\end{array}\right. \\
& \begin{cases}\theta_{i} k_{i}=k_{i}^{-1} & \\
\theta_{i} k_{j}=k_{i} k_{j} & \text { if } a_{i j}=-1 \\
\theta_{i} k_{j}=k_{j} & \text { if } a_{i j}=0\end{cases}
\end{aligned}
$$

which satisfies the braid relations:

$$
\begin{array}{cll}
\theta_{i} \theta_{j} \theta_{i}=\theta_{j} \theta_{i} \theta_{j} & \text { if } & a_{i j}=-1 \\
\theta_{i} \theta_{j}=\theta_{j} \theta_{i} & \text { if } & a_{i j}=0 .
\end{array}
$$

Proof. The proof follows by a straightforward calculation. First one has to show that the $\theta_{i}$ are really automorphisms, that is one has to verify that the commutator $\left[e_{i}, f_{j}\right]$ is invariant under the action of the $\theta_{i}$. We have

$$
\begin{aligned}
\theta_{i}\left(e_{i} f_{i}-f_{i} e_{i}\right) & =f_{i} k_{i}^{2} k_{i}^{-2} e_{i}-k_{i}^{-2} e_{i} f_{i} k_{i}^{2} \\
& =\left(f_{i} e_{i}-e_{i} f_{i}\right) \\
& =\frac{k_{i}-k_{i}^{-1}}{q^{2}-q^{-2}} .
\end{aligned}
$$

A similar calculation shows that if $a_{i j}=-1$

$$
\theta_{i}\left(e_{j} f_{j}-f_{j} e_{j}\right)=\frac{k_{i} k_{j}-k_{i}^{-1} k_{j}^{-1}}{q^{2}-q^{-2}} \text { if } a_{i j}=-1
$$

To check the rest of the relations is done by similar calculations. It is straightforward to verify that the definition of the action of the $\theta_{i}$ on the generators imply the braid relations (3.24).

\subsection{Quantization of the Poisson Brackets for $W$ Algebras}

In this subsection we show that a modification of the Miura transformation can be used to define the quantization of the Poisson brackets introduced in Sect. 2. This modification turns out to be related to the vertex realization of Kac--Moody algebras. By introducing so-called $\tau$-functions we will be able to define the quantization of the Poisson brackets for $W$ algebras.

Subsequently we use the relation between vertex representations of Kac-Moody algebras and $W$ algebras, provided by the Miura transformation, to study decompositions of vertex representations of Kac-Moody algebras into irreducible representations. It is known that under certain conditions these decompositions comprise only a finite number of irreducible representations. We will find an 
alternative explanation for this phenomenon based on the Miura transformation. As we will show this finiteness property is related to properties of the representation theory of the quantum group associated with the Kac-Moody algebra in the case $q$ is a root of unity.

In order to introduce these $\tau$-functions we need some notation. Let $V\left(\Lambda_{k}\right)$ be the vertex representation of $A_{N}^{(1)}$ associated with the Dynkin vertex $c_{k}$ corresponding to the highest weight vector $v_{k}$. $\Lambda$ is defined as the sum over the exponents as in Sect. 2. As is well known, this provides a representation on differential polynomials in infinitely many variables on which the algebra acts by differential operators of the form (2.45). Furthermore, on $V\left(\Lambda_{k}\right)$ there is a hermitian form $(\cdot, \cdot)$ which is expressed in terms of differential polynomials. Denote by $V^{\perp}\left(2 \Lambda_{k}\right)$ the orthogonal complement of $V\left(2 \Lambda_{k}\right)$ with respect to this hermitian form. The $\tau_{k}$-function is then introduced as the element in $V\left(\Lambda_{k}\right)$ which solves the differential equation

$$
(f, \tau \otimes \tau)=0 \text { for all } f \in V^{\perp}\left(2 \Lambda_{k}\right)
$$

such that $\tau \otimes \tau \in V\left(2 \Lambda_{k}\right) \subset V\left(\Lambda_{k}\right) \otimes V\left(\Lambda_{k}\right)$. Equation (3.16) is a bilinear differential equation in $\tau$ called the Hirota equation (see e.g. [34]).

A well known theorem in [35] states that the $\tau$-function is in the group orbit of the highest weight vector $v_{k}$ (recall that the highest weight representation is integrable, i.e. locally nilpotent so it can be exponentiated to give a group action on $v_{k}$ ):

$$
\tau_{k}=c g v_{k},
$$

where $c$ is an arbitrary complex constant and $g$ is an element of the Kac-Moody group. Observe that the group element $g$ does not determine $\tau_{k}$ uniquely since conjugation by unipotent elements leaves $\tau_{k}$ invariant. The relation with the $\tau$-function (and its associated bilinear equation) with the modified (generalized) $\mathrm{KdV}$-hierarchy is extensively discussed in [33]. We will not discuss this formalism here. ${ }^{1}$ Using the results obtained there one finds that

$$
v=\partial_{x} \Psi \Psi^{-1}-\Lambda
$$

provides a solution of the modified $\mathrm{KdV}$ hierarchy. The function $\Psi$ is defined on the subspace $\Gamma_{+}$in the notation of [33]. From this it follows that the bilinear equation satisfied by the $\tau$-function is equivalent to the nonlinear modified $\mathrm{KdV}$ hierarchy. We will now show that the Miura transformation applied to the bilinear equation is related to the coset construction by which one can define the quantization of the $W$ algebra. Let $V\left(\Lambda_{0}\right)$ and $V\left(\Lambda_{1}\right)$ be the two fundamental $A_{1}^{(1)}$ representations in the vertex realization and put

$$
V=V\left(\Lambda_{0}\right) \otimes V\left(\Lambda_{1}\right)
$$

we now have the following

${ }^{1}$ The relation between $\tau$-functions and (irreducible) representations of the Virasoro and $W$-algebras has been considered also in [49] 
Theorem 3.5. The action of $A_{(1)}^{(1)} \otimes A_{N}^{(1)}$ in $\sum V_{k}$, where we put

$$
V_{2 k} \simeq V\left(\Lambda_{0}\right), \quad V_{2 k+1} \simeq V\left(\Lambda_{1}\right)
$$

is decomposed into irreducible representations with highest weight vectors $1 \otimes v_{k}$ of $A_{1}^{(1)} k \in \mathbf{Z}^{+}$. In particular for $N<\infty$ the number of irreducible components is finite.

Proof. Consider the modified $\mathrm{KdV}$ solution

$$
v=\partial \log \left(\tau_{0} / \tau_{1}\right)
$$

and apply the Miura transformation. We have to be careful here: since we started with a highest weight representation of the Kac-Moody algebra, we have to make sure that the resulting representation of the Virasoro algebra has a highest weight too. This is accomplished by imposing a normal ordering description:

$$
L=: \prod_{i} \partial-v_{i}:=\sum_{i} W_{i} \partial^{i}
$$

where the coefficients $W_{i}$ are the normal ordered expressions

$$
\begin{aligned}
W_{0} & =1, \\
u_{1}=W_{1} & =v^{\prime}-: v^{2}: \Leftrightarrow \\
L_{n} & =(n-1) a_{n}+\sum_{n} a_{n} a_{m-n}-n \theta_{n+m} \theta(n),
\end{aligned}
$$

where $\theta(n)=1$ if $n>0$ and zero otherwise. Thus we have afforded a highest weight representation of the Virasoro algebra in the vertex realization of $V\left(\Lambda_{0}\right)$. If we now identify $u(x)$ with the energy momentum tensor $T$ generating the conformal transformations, then the second Hamilton operator associated with the Lax equation for $L=\partial^{2}+u$ is with (3.31) equivalent to the operator product expansion

$$
T(x) T(y) \propto \frac{c / 4}{(x-y)^{4}}+\frac{T(x)+T(y)}{(x-y)^{2}}+\text { regular terms. }
$$

This is in fact precisely what happens in the so-called coset construction of $[6,7]$. Indeed direct calculation of the possible highest weights yields the finite irreducibility.

Using the higher order Miura transformations one may generalize to the following

Theorem 3.6. Let $V\left(2 \Lambda_{0}\right)$ be the $A_{M}^{(1)}$ vertex realization generated by $1 \otimes 1$, which affords the space for the bilinear equation for the generalized modified $\mathrm{KdV}$ hierarchy. Let $V=\sum_{k} V_{k}$, where $V_{k}$ denotes a fundamental representation of $A_{M}^{(1)}$. Then the action of $A_{M}^{(1)} \otimes A_{N}^{(1)}$ on $V$ for $N<\infty$ breaks up into finitely many irreducible representations of $A_{M}^{(1)}$ induced by the Miura transformation of order $M$ with the normal ordering prescription as given in (3.31).

The higher order Miura transformations thus provide highest weight representation of the $W$ algebra associated with $A_{M}^{(1)}$. However the identification with an operator product expansion is more subtle. For example in the case $M=2$ (i.e. $S L_{3}$ ), one finds that the operator product expansion corresponding to the 
quantization of the bracket $(2.72)$ reads

$$
\begin{gathered}
v(x) v(y) \propto \frac{c / 3}{(x-y)^{6}}+\frac{2 T}{(x-y)^{4}}+\frac{T^{\prime}}{(x-y)^{3}}+\frac{1}{(x-y)^{2}}\left(\frac{5}{18} T^{\prime \prime}+\frac{2}{9} u^{2}+\frac{1}{6} \alpha\right) \\
\frac{1}{(x-y)}\left(\frac{1}{18} T^{\prime \prime \prime}+\frac{1}{9}\left(u^{2}\right)^{\prime}+\frac{1}{18} \alpha+\frac{1}{36} \alpha^{\prime}\right),
\end{gathered}
$$

where

$$
\begin{aligned}
& \alpha=12\left(\left(\frac{16}{22+5 c}-\frac{1}{9}\right) \Lambda+\frac{1}{90} T^{\prime \prime}\right), \\
& \Lambda=: T^{2}:-\frac{3}{10} T^{\prime \prime} .
\end{aligned}
$$

In these formulae we have made the identification of $u$ and $T$ and used the fact that the second Hamilton operator (2.76) is symmetric. To identify the operator $u^{2}$ we may use the Miura transformation also. It then follows that $u^{2}$ transforms as a spin-4 operator, hence similar as $\Lambda$ and contrary to : $T^{2}:$. If we make the identification of $u^{2}$ with $\Lambda$ then (3.33) is in agreement with [7]. The fact that the above operator product expansions indeed defines closed algebras (with quadratic terms) is a direct consequence of the property that the Miura transformation is a Hamilton transformation for the second Hamilton structure which defines a closed algebra.

It is interesting to consider the limit $N \rightarrow \infty$ with respect to the bilinear equation for the $\tau$-function. The bilinear equation (3.26) for $A_{N}^{(1)}$ has the general form [33]

$$
\left.P_{i}\left(\partial_{y}\right) \tau(x-y) \tau(x+y)\right|_{y=0}=0, \quad i=1,2,3, \ldots,
$$

where $x$ and $y, x=\left(x_{1}, \ldots, x_{n-1}, x_{n+1}, \ldots x_{2 n-1}, x_{2 n+1}, \ldots\right)$, and similar for $y$, are vectors associated with the two copies of the vertex representations $V\left(\Lambda_{0}\right)$ and $P_{i}$ is a certain differential polynomial in "time" variables $y_{j}$. Their explicit construction can be found in [34] for $A_{N}^{(1)}$. Using this it is easy to prove that in the limit $N \rightarrow \infty$ Theorem (3.5) implies that all the polynomials become linearly independent. So one obtains the Virasoro algebra in this case entirely from $A_{\infty}$. The hierarchy associated to the bilinear equation in this limit is known as the K.P. hierarchy. If $N<\infty$ it follows easily that the polynomials are not all linearly independent. One can now choose a suitable set of coordinates such that the $\tau$-function does not depend on $x_{n}, x_{2 n}, x_{3 n}, \ldots$. So the bilinear equation breaks up into "irreducible" components. The nontrivial part is equivalent to the generalized $\mathrm{KdV}$ hierarchies for $A_{N}^{(1)}$, while the trivial part, corresponding to a trivial hierarchy, decouples. The linear independence of the polynomials $P_{i}$ in the case $N=\infty$ implies that in Theorem (3.5) all the irreducible representations of the Virasoro algebra appear. One can consider the same limit in Theorem (3.6). Without knowing the precise form of the analogue of the bilinear equation one may still conclude that also in this case all irreducible representations will appear.

Summarizing we conclude that for finite $N$ Theorem (3.5) respectively its generalization (3.6) provide reducible representations of the Virasoro algebra respectively of a $W$ algebra, provided by hierarchies of partial differential equations, 
which decompose into a finite linear combination of irreducible representations. This is similar to the situation in the well known coset construction applied to higher level $k$ representations, i.e. to $A_{N}^{(1)} \otimes A_{N}^{(k)} \subset A_{N}^{(k+1)}$ as described in [7]. It is illuminating to explore this relation further, using the Miura transformation, to describe the apparent connection with Kac-Moody current algebras. As a result we will be able to relate the central charge with the $q$ values obtained in the previous section. We will first discuss this for the Virasoro case, i.e. for Theorem (3.4). The generalization is straightforward.

Recall from Sect. 2.1 that the variable $u(x)$ transformed as the energy momentum tensor. In view of the Miura transformation (3.21) one thus has two independent solutions $v_{a}(x)$ which can be interpreted as currents $i \partial_{a} \phi$. These currents build up the level $k S L_{2}$ Kac-Moody algebra. Via the Sugawara construction this leads to the usual $S L_{2}$ level $k$ energy momentum tensor:

$$
T_{S L_{2}}=\frac{1}{k+2} \sum: v^{2}: .
$$

In this respect we conclude that the Miura transformation has the effect of "adding a electric charge at infinity" to this energy momentum tensor. Computing the operator product expansion (3.32), one finds for the central charge:

$$
c_{S L_{2}}^{(k)}=\frac{3 k}{k+2}-6 k
$$

which is in agreement with the value found in $[39,36,8]$. At this point we recall the value of the central charge $c$ of an irreducible representation of the Virasoro algebra provided by the coset construction as explained in Theorem 3.4:

$$
c=13-\frac{6}{k+2}-6(k+2)=c_{S L_{2}}^{(k)}-2
$$

We thus observe that the difference is independent of the level. (This observation lies at the basis of a cohomological argument in [51], showing that the cohomology of the reduced phase space is isomorphic to irreducible representations of the Virasoro algebra.) Combining with the remarks above, we find that the irreducible representations of the Virasoro algebra provided by the Miura transformation (3.21) have central charge $c$ given by (3.27). This result is easily generalized to $W$-algebras corresponding to $S L_{N}$. The central charge of the usual energy momentum tensor for a $S L_{N}$ level $K$ Kac-Moody algebra reads $k\left(n^{2}-1\right) /(k+n)$. Applying the Miura transformation one finds in a similar way as above the modification of this value of the central charge:

$$
c_{S L_{N}}^{(k)}=N^{4}-N\left(N^{2}-1\right)\left(\frac{1}{k+N}+k+N\right)-1 .
$$

The central charge of the quantum $W$ algebra corresponding to $S L_{N}$ has been computed in [7]. Using the result found there, one finds the generalization of (3.37),

$$
c_{W}=c_{S L_{N}}^{(k)}-\left(N^{4}-2 N^{3}+N\right) .
$$


Again we observe that the difference is independent of the level $k$. We will find a geometrical explanation of this fact in the next subsection. Let us conclude this subsection with a comment on the relation with the value of the deformation parameter $q$. This relation follows for example by applying the so-called Bathe-construction [37] to the representations of the $W$ algebras obtained above. We will not present this formalism here. Using it, it follows that when $q$ is a root of unity it depends on the level $k$ via

$$
q=e^{2 \pi i / k+N},
$$

as is shown in [42]. In fact this formula was derived much earlier in [16]. This concludes our discussion on the relation between the quantum group and the quantum conformal algebras.

3.3 The Action of a Quantum W Algebra on the Moduli Space of Riemann Surfaces. In this last subsection we will find a geometrical explanation for the relation between the representations of $W$ algebras and representations of Kac-Moody current algebras, provided by the Miura transformation (3.21). The key point is to find a geometrical interpretation for the Hillbert space associated with the quantum group introduced in Sect. (3.1). For this it is convenient to consider the quantum $R$ matrix as the monodromy matrix of the correlation function associated with the fields $\phi$ in the underlying conformal field theory. This is extensively discussed in $[42,43]$. An $n$ point correlation function $G\left(x_{1}, \ldots, x_{n}\right)$ defines a multivalued function on a subspace $H_{0} \subset \mathbf{C}^{n}$ as follows [43]. Introduce first the set $H$ :

$$
H=\left\{\left(x_{1}, x_{2}, \ldots, x_{n}\right) \in \mathbf{C}^{n} \mid x_{i} \neq x_{j} \text { for all } i \neq j\right\} .
$$

The correlation functions are actually invariant under a permutation of the points $x_{i} \in H$, hence they are invariant under the-diagonal action of the symmetric group $S_{n}$. That is the correlation functions are multi-valued functions on

$$
H_{0}=H / S_{n} \text {. }
$$

It is not hard to see that $H_{0}$ is not simply connected, and in fact:

$$
\pi_{1}\left(H_{0}\right)=B_{n} \text {. }
$$

This shows that there is a natural diagonal action of the braid group on the correlation functions. One can use this action to define the correlation functions as single valued functions on the universal covering of $H_{0}$. Consider two curves $\xi_{i}(t), \xi_{i+1}(t)$ with $t \in[0,1]$ such that their composition represents a loop in $\mathbf{C}^{2}$,

$$
\begin{aligned}
\xi_{i}(0) & =x_{i} & \xi_{i}(1) & =x_{i+1}, \\
\xi_{i+1}(0) & =x_{i+1} & \xi_{i+1}(1) & =x_{i} .
\end{aligned}
$$

Restricting to $H_{0}$ this defines an element $R_{i i+1} \in B_{n}$. In fact by acting with this element on $G\left(x_{1}, \ldots, x_{n}\right)$ defines a new correlation function on $R_{i i+1} H_{0}$. Repeating this $n$ times one produces a correlation function which only depends on a given element $R \in B_{n}$ :

$$
R=\prod_{i=0}^{n-1} R_{i i+1}
$$


such that

$$
G\left(x_{1}, \ldots, x_{n}\right)=R G\left(\xi_{1}, \ldots, \xi_{n}\right)
$$

defines a single valued function on the universal cover $R H_{0}$ of $H_{0}$. This shows that the correlation functions define a representation of the braid group, which determines completely the monodromy of these functions:

$$
G\left(\xi_{1}(0), \ldots, \xi_{n}(0)\right)=R G\left(\xi_{1}(1), \ldots, \xi_{n}(1)\right) .
$$

Observe that the monodromy of $G$ is trivial if and only if $R$ defines a unitrary representation of $B_{n}$. Equation (3.41) shows that correlation functions are actually sections of a complex vector bundle $V$ over $H_{0}$ with fibre $V_{1} \otimes \cdots \otimes V_{n}, V_{i}=\mathbf{C}^{m}$.

The requirement that the monodromy is trivial is thus equivalent with requiring that $R$ is the holonomy of a (projectively) flat connection in $V$. (The projectivity of the connection will become clear below.) Locally such a connection is trivial while globally it corresponds to a homomorphism

$$
\pi_{1}\left(H_{0}\right) \rightarrow G
$$

which describes its holonomy. Here $G$ is some Lie group acting in End $(V)$ which we will specify below. Thus, we find that the quantum $R$ matrix amounts to a monodromy representation of $\pi_{1}\left(H_{0}\right)$. We can identify this holonomy quite easily by using the relation between representations of the braid group and of the mapping class group of a Riemann surface. It is well known [22] that the mapping class group $\Gamma_{n}$ of a genus $n$ compact Riemann surface defined as

$$
\Gamma_{n}=\operatorname{Diff} / \operatorname{Diff}_{0},
$$

is generated by the Dehn twists along $2 n+1$ nonintersecting homology cycles of the surface and one further element, describing the twist along an intersecting cycle. (Such a cycle is only present when the genus is greater than 1.) The Dehn twists satisfy the relations of a $2 n+2$ string braid group. So the mapping class group of a genus $n-1$ Riemann surface $\Sigma$ is generated by a homomorphic image of $B_{2 n+2}$ together with one extra element if the genus is larger than one. So on the sphere and on the torus the complex vector bundle admits a flat connection, while for higher genus it admits only a projectively flat connection. Thus the representation (3.34) is equivalent with the monodromy representation of the fundamental group of $\Sigma$,

$$
\pi_{1}(\Sigma) \rightarrow G
$$

These observations give rise to an alternative description of the representation space of the quantum group associated with $R$. Namely it corresponds to the space of functions on the possible monodromy representations (3.35). More precisely, if $V$ denotes the Hilbert space mentioned in Theorem (3.2) we have

$$
V=\operatorname{Hom}\left(\pi_{1}(\Sigma), G\right) / G
$$

where Hom denotes the set of representations $\pi_{1}(\Sigma) \rightarrow G$. The Hilbert space is obtained as this set modulo the action of the inner automorphisms of $G$. It is well known that the smooth part of this space is a manifold which admits a Kähler 
structure, which depends on the choice of $G$. In particular in [44] it is shown that if $G=S L_{2}$ then this Kähler structure can be identified as the Weil-Petersson Kähler form on the moduli space of stable Riemann surfaces. One may generalize this to other groups; here we will consider the case for the groups $S L_{N}$ (see [44]). For definiteness we first consider $G=S L_{2}$. Thus, the space $V$ in (3.46) should provide an irreducible representation of the Virasoro algebra. We will now show that this is indeed the case. The argument is as follows. The Kähler form is in fact the first Chern class on the determinant line bundle $\lambda_{j}$ of holomorphic $j$ differentials on moduli space. In [41] we have computed this Chern class as the curvature of the so-called Quillen metric. The result found there reads:

$$
c_{1}\left(\lambda_{j}\right)=\left(\frac{6 j(j-1)+1}{24 \pi^{2}}\right) \omega_{\mathrm{wp}},
$$

where $\omega_{\text {wp }}$ is the Weil-Peterson Kähler from.

Next we recall the result obtained in [1], namely that a given representation of the Virasoro algebra with central charge $c$, corresponds to the space of holomorphic sections of a determinant line bundle $L_{c}$. This space is endowed with a Hermitian metric. By a theorem of Harer this bundle is isomorphic to a power of the determinant line bundle of the holomorphic one forms. More precisely: $L_{c}^{c / 2} \simeq \lambda_{1}$. Thus it follows that the curvature denoted by $\Omega$ of the Hermitian metric on $L_{c}$ is proportional to the Weil-Petersson Kähler form:

$$
\frac{c}{2} \Omega=\frac{1}{24 \pi^{2}} \omega_{\mathrm{wp}}
$$

At this point we recall that the value of the central charge $c$ is given by (3.27). The bundle $\lambda_{j}$ together with its anti-holomorphic counterpart, corresponds to the Hilbert space of a $(b, c)$ ghost system of weight $(j-1, j)$, which has a so-called conformal anomaly given by $-2\left(6^{2 j^{2}}-6 j+1\right)$. So we arrive at the following geometrical interpretation of the representations obtained in the previous subsection, namely that the Hilbert space $L_{c}$ (providing an irreducible representation of the Virasoro algebra) is isomorphic to an irreducible representation of the $S L_{2}$ level $k$ current algebra after application of the Miura transformation, times the Hilbert space of a $(b, c)$ ghost system of spin $j=1$.

It seems natural to expect that this generalizes to the $W$ algebras for $S L_{N}$. The following remarks sustain this idea. For $S L_{N}$ the factor $N^{4}-2 N^{3}+N$ corresponds to the sum of the conformal anomalies of $n$ different $(b, c)$ ghost systems, for $1 \leqq n \leqq N-1$, of weight $(-N+n-1, N-n)$. The fact all of them have to be taken into account is consistent with the filtration property of the $W$ algebras discussed in Sect. (2.2). Furthermore, one expects that the anomalies cancel for a particular value of $c$, i.e. there should be a global section on the moduli space, like for the Virasoro algebra when $c=26$. In this case there is an invariant of the action of the Virasoro algebra given by the so-called Mumford form:

$$
\mu^{(1)}=\lambda_{2} \otimes \lambda_{0}^{-13} \text {. }
$$


In a possible generalization to the other $W$ algebras the filtration property of these algebras may be used to decompose the general bundle $V$ into subbundles $V=V_{1} \otimes \cdots \otimes V_{N}$ where each of the subbundles $V_{i}$ provide irreducible representations of the lower order $W$ algebras. Subsequently, using the Grothendieck-Riemann-Roch theorem one may determine the invariant action on the moduli space. For example for the $S L_{3} W$ algebra this suggests the invariant

$$
\mu^{(2)}=\lambda_{3} \otimes \lambda_{2} \otimes \lambda_{0}^{-13-37}
$$

so that the critical value of the central charge is given by $c=100$. This value also appears in [7] where it is related to the $(b, c)$ ghost systems of weight $(0,1)$ and $(-1,2)$. This concludes our discussion on the $W$ algebra acting on the moduli space.

Note added. During the typing of the manuscript recent work by P. Mathieu [45] and K. Yamagishi [46] came to our attention, in which similar aspects as discussed in Sects. 2 and 3 have been presented. We also received a preprint by G. Lusztig [47], in which a proof is presented of the quantization of integrable irreducible highest weight modules of arbitrary Kac-Moody algebras. The contents of the present paper have been reported at the CIME conference on "Global Geometry and Mathematical Physics," held at Montecatini, Italy, July 1988.

Acknowledgements. I wish to thank L. Alvarez-Gaumé, B. de Wit and M. Hazewinkel for their useful remarks and reading early versions of the manuscript and also for bringing reference [47] to my attention. I benefitted much from discussions with F. A. Bais, H.-J. Imbens, F. Nijhoff, K. H. Rehren and K. Schoutens. This work has been supported financially by "Stichting Fundamenteel Onderzoek der Materie," F.O.M.

\section{References}

1. Friedan, D., Shenker, S.: The analytic geometry of $d=2$ conformal field theory. Nucl. Phys. B281, 50 (1987)

2. Arbarello, E., De Concini, C., Kac, V., Procesi, C.: Moduli spaces of curves and representation theory. Commun. Math. Phys. 117, 1 (1988)

3. Alvarez-Gaumé, L., Gomez, C., Reina, C.: Loop groups Grassmannians and string theory. Phys. Lett. 109B, 55 (1987)

4. Alvarez-Gaumé, L., Gomez, C., More, G., Vafa, C.: Strings in the operator formalism. Nucl. Phys. B303, 455 (1988)

5. Zamolodchikov, A. B.: Infinite additional symmetries in two dimensional quantum conformal field theory. Theor. Math. Phys. 65, 1205 (1985)

6. Goddard, P., Kent, A., Olive, D.: Phys. Lett. 152B, 88 (1985); Commun. Math. Phys. 103, 105(1985)

7. Bais, F.A., Bouwknegt, P., Surridge, M., Schoutens, K.: Extensions of the Virasoro algebra constructed from Kac-Moody algebras using higher order Casimir invariants. Nucl. Phys. B304, 348 (1988); Coset construction for extended Virasoro algebras. Nucl. Phys. B304, 371 (1988)

8. Fateev, V., Lykyanov, S.: The models of two dimensional quantum field theory with $Z_{N}$-symmetry. Int. J. Mod. Phys. 3, 507 (1988)

9. Bilal, A., Gervais, J.: A systematic approach to conformal systems with extended Virasoro symmetries. Phys. Lett. B206, 412 (1988)

10. Beilinson, A., Manin, Yu., Schechtman, V.: Sheaves of the Virasoro and Neveu-Schwarz algebra. In: K-Theory, arithmetic and geometry. Manin Yu. (ed.) Lecture Notes in Mathematics vol. 1289. Berlin, Heidelberg, New York: Springer 1987

11. Drinfeld, V. G.: Quantum groups. In: Proceedings of the I.C.M. Berkeley (1986)

12. Drinfeld: V. G.: Hamiltonian structures on Lie groups, Lie bialgebras and the geometrical meaning of the classical Yang-Baxter equation. Sov. Math. Dokl. 268, 285 (1983) 
13. Faddeev, L.: Integrable models in (1 +1$)$-dimensional quantum field theory. In: Procedings of Les Houches 1982, vol. 561. Stora, R., Zuber, J.-B. (eds.) Amsterdam: North Holland 1989

14. Drinfeld, V. G., Sokolov, V. V.: Lie algebras and equations of Korteweg de Vries type. J. Sov. Math. 30, 1975 (1985)

15. Harer, J.: The second homology group of the mapping class group of an orientable surface. Invent. Math. 72, 221 (1983)

16. Gervais, J.-L.: Infinite family of polynomial functions of the Virasoro generators with vanishing Poisson brackets. Phys. Lett. 160B, 277 (1985)

17. Kupershmidt, B. A.: A super Korteweg de Vries equation: An integrable system. Phys. Lett. 102A, 213 (1984); Super Korteweg de Vries equations associated to the super extensions of the Virasoro algebra. Phys. Lett. 109A, 417 (1985)

18. Kupershmidt, B. A., Wilson, G.: Modifying Lax equations and the second Hamiltonian structure. Invent. Math. 62, 403 (1981)

19. Jimbo, M.: A $q$-analogue of $U(N+1)$, Hecke algebra and the Yang-Baxter equation. Lett. Math. Phys. 11, 247 (1986)

20. Jones, V.: Hecke algebra representations of braid groups and link polynomials. Ann. Math. 126, 335 (1987)

21. Wenzl, H.: Hecke algebras of type $A_{n}$ and subfactors. Ph.D. Thesis Univ. Penn.

22. Birman, J.: Braids, links and mapping class groups. Ann. Math. Stud. 82 (1974)

23. Kirillov, A. A.: Orbits of the group of diffeomorphisms of a circle and local Lie-super algebras. Funct. Anal. Appl. 15, 135 (1981)

24. Segal, G.: Unitary representations of some infinite dimensional groups. Commun. Math. Phys. 80, 307 (1981)

25. Witten, E.: Coadjoint orbits of the Virasoro group. Commun. Math. Phys. 114, 1 (1988)

26. Kirillov, A. A.: Elements of the theory of representations, Sect. 15. Berlin, Heidelberg, New York: Springer 1976

27. Gelfand, I. A., Dickey, L. A.: Fractional powers of operators and Hamiltonian systems. Funct. Anal. Appl. 10, 4(1976); The Resolvent and Hamiltonian systems. Funct. Anal. Appl. 11, 93 (1977)

28. Gelfand, I. M., Dorfman, I. Ya: Hamiltonian operators and infinite dimensional Lie algebras. Funct. Anal. Appl. 15, 173 (1982)

29. Manin, Yu.: Algebraic aspects of nonlinear differential equations. J. Sov. Math. 11, 1 (1979)

30. Reyman, A. G., Semenov-Tian-Shansky, M.: Current algebras and nonlinear partial differential equations. Sov. Math. Dokl. 21, 630 (1980)

31. Sklyanin, E. K.: Some algebraic structures connected with the Yang-Baxter equation. Furrct. Anal. Appl. 16, 263 (1983)

32. Faddeev, L. D., Reshetikhin, N., Takhtajan, L.: Quantization of Lie groups and Lie algebras, LOMI preprint E-14-87, Leningrad 1987

33. Pressley, A., Segal, G.: Loop Groups. Oxford: Clarendon Press 1986

34. Kac, V. G.: Infinite dimensional Lie algebras, C.U.P. (1985)

35. Kac, V. G., Peterson, D. H.: Regular functions on certain infinite dimensional groups. Artin, M., Tate, J. (eds). In: Arithmetic and Geometry, 141 (1983)

36. Pasquier, V.: Continuum limit of lattice models built on quantum groups, Saclay preprint SPhT/87-125; Etiology of IRF models, Saclay preprint SPhT/88-20

37. de Vega, H. J., Karowski, M.: Nucl. Phys. B[FS19], 619 (1987)

38. Atiyah, M. F., Bott. R.: The Yang-Mills equations over Riemann surfaces. Phil. Trans. R. Soc. Lond. A 308, 523 (1982)

39. Belavin, A. A., Polyakov, A., Zamolodchikov, A. B.: Infinite conformal symmetry in two dimensional conformal field theory. Nucl. Phys. B241, 333 (1984)

40. Belinson, A., Manin, Yu.: The Mumford form and Polyakov measure in string theory. Commun. Math. Phys. 107, 359 (1986)

41. Smit, D.-J.: String theory and the algebraic geometry of moduli spaces. Commun. Math. Phys. 114, $645(1988)$

42. Kohno, T.: Monodromy representations of braid groups and Yang-Baxter equations. Ann. Inst. Fourier, 37, 139 (1987)

43. Fröhlich, J.: Statistics of fields, the Yang-Baxter equation, and the theory of knots and links, Zürich preprint 1987 
44. Goldman, W.: The symplectic nature of fundamental groups of surfaces. Adv. Math. 54, 200 (1984)

45. Mathieu, P.: Extended classical conformal algebras and the second Hamiltonian structure of Lax equations. Phys. Lett. B208, 101 (1988)

46. Yamagishi, K.: The KP hierarchy and extended Virasoro algebras. Phys. Lett. B205, 466 (1988)

47. Lusztig, G.: Quantum deformations of certain simple modules over universal enveloping algebras. MIT Dept. Mathematics preprint 1988

48. Lykyanov, S., Fateev, V.: Additional symmetries in two dimensional conformal field theory and exactly solvable models, Parts I, II, III. Institute for Theoretical Physics, Kiev preprint ITP-88-74P

49. Bais, F. A., de Vos, K.: Private communication, and: preprint (to appear)

50. Bakas, I.: Hamiltonian reduction and conformal symmetries in two dimensions, University of Texas, preprint November 1988

51. Bershadsky, M., Ooguri, H.: Hidden $S L_{n}$ symmetries in conformal field theories, IASSNS preprint $89 / 09$

Communicated by L. Alvarez-Gaumé

Received September 16, 1988; in revised form May 12, 1989 
